\title{
Keep off the grass? Cannabis, cognition and addiction
}

\author{
H. Valerie Curran*1, Tom P. Freeman ${ }^{1}$, Claire Mokrysz ${ }^{1}$, David A. Lewis ${ }^{2}$,
}

\author{
Celia J. A. Morgan ${ }^{1,3}$ \& Loren H. Parsons ${ }^{4}$
}

\footnotetext{
${ }^{1}$ Clinical Psychopharmacology Unit, University College London, Gower St, London WC1E 6BT, UK.

${ }^{2}$ Department of Psychiatry, University of Pittsburgh, 3811 O'Hara St, Pittsburgh PA 15213, USA.

${ }^{3}$ Psychopharmacology and Addiction Research Centre, University of Exeter, Perry Road, Exeter EX4 4QG, UK.

${ }^{4}$ The Scripps Research Institute, 10550 N. Torrey Pines Road, SP30-2001, La Jolla, USA.

*Correspondence to: H.V.C., v.curran@ucl.ac.uk
}

\begin{abstract}
In an increasing number of states and countries, cannabis now stands poised to join alcohol and tobacco as a legal drug. Quantifying the relative adverse and beneficial effects of cannabis and its constituent cannabinoids should therefore be prioritised. Whereas newspaper headlines have focused on links between cannabis and psychosis, less attention has been paid to the much more common problem of cannabis addiction. Certain cognitive changes have also been attributed to cannabis use, although their causality and longevity are fiercely debated. Identifying why some individuals are more vulnerable than others to the adverse effects of cannabis is now of paramount importance to public health. Here we review the current state of knowledge about such vulnerability factors, the variations in types of cannabis, and their relationship to cognition and addiction.
\end{abstract}

\section{Introduction}


For millennia, cannabis has been used medically and for religious purposes, most notably in China and India. The plant and its many constituent cannabinoids are now becoming increasingly important in modern medicine, particularly in the treatment of chronic pain and spasticity $^{1}$. A much more widespread global use is for pleasure ${ }^{2}$ : the 'stoned' experience varies widely across individuals but often includes euphoria, a heightened awareness of music and colour, and the tendencies to eat a lot and to giggle profusely ${ }^{2}$. Despite its pleasurable effects, most scientific research has focused on adverse consequences of using the drug, such as addiction, cognitive impairment and possible increased risk of psychotic illness ${ }^{3,4}$.

We do not know how patterns of use will change as legalisation proliferates, but even a small percentage increase in the current 182 million users worldwide ${ }^{5}$ will mean a considerable surge in absolute numbers. Are we now able to use existing evidence about the less desirable effects of cannabis use to help us to look forward to the future?

This article aims to survey our current state of knowledge about the effects of cannabis and then pinpoint how we should be increasing our understanding of the effects of cannabis, given its potentially soaring future use. We first summarise the variety of unique ingredients in cannabis and outline how its use affects cognition, learning and memory. We survey evidence of how the effects of the drug vary according to the maturational state of the brain and then go on to discuss cannabis addiction and the mental health problems that are often related to it. Finally, we identify the important gaps in our current knowledge and look to the future in terms of both research and the current tide of changes to the legislation of cannabis.

\section{Cannabis: a plant with many forms}

Purple Haze, Northern Lights, charas, skunk, resin, grass, marijuana, weed... The multitude of names for cannabis in part reflects variations in genetics, growing conditions, processing, and 
constituent cannabinoids and terpenoids in different strains of the plant. Of the roughly 100 unique ingredients in cannabis that are called cannabinoids, most research to date has focused on the two most prominent of these: delta-9-tetrahydrocannabinol $\left(\Delta^{9}\right.$-THC) and cannabidiol (CBD). These two compounds appear to have a range of opposing effects on the human brain and behaviour. For example, $\Delta^{9}$-THC acutely impairs learning, produces psychosis-like effects and increases anxiety ${ }^{6}$, whereas CBD can enhance learning ${ }^{7}$ and has antipsychotic ${ }^{8}$ and antianxiety ${ }^{9}$ properties in humans. When taken together, CBD may ameliorate the harmful effects of $\Delta^{9}-\mathrm{THC}^{10,11}$.

$\Delta^{9}$-THC acts as a partial agonist at cannabinoid $\mathrm{CB}_{1}$ receptors $(\mathrm{CB} 1 \mathrm{Rs})$, whereas CBD has a complex range of pharmacological actions. For example, although CBD has low affinity for $\mathrm{CB}_{1} \mathrm{R}$ it can attenuate $\mathrm{CB}_{1}$ agonist effects in brain even at low concentrations (e.g. providing functional antagonism of $\mathrm{CB}_{1} \mathrm{R}$ signaling $)^{12}$. Conversely, $\mathrm{CBD}$ reduces the cellular reuptake and hydrolysis of the endogenous cannabinoid anandamide (AEA) in brain ${ }^{12,13}$ Neuroimaging studies have documented opposing effects of $\Delta^{9}$-THC and CBD on blood oxygenation leveldependent (BOLD) signal during performance of several cognitive and emotional tasks, including striatal response during memory retrieval and amygdala response to fearful faces ${ }^{14}$.

Over the past two decades, the $\Delta^{9}$-THC content of street cannabis has risen dramatically, whereas its CBD content has decreased to negligible levels. For example, in the United States, the $\Delta^{9}$-THC content of street cannabis rose from $4 \%$ in 1995 to $12 \%$ in $2014^{15}$. In Europe 1617 and Australia ${ }^{18}$, high-potency cannabis containing $~ 15 \% \Delta^{9}$-THC and less than $0.1 \%$ CBD now dominates the market. Thus, the type of cannabis available years ago differs considerably from that sold today, limiting the relevance of older longitudinal cohort studies (for example, the New Zealand Birth Cohort study, see Box 1) to the mental health and cognitive function of contemporary users. In the United States, the cannabis that the National Institute of Drug Abuse 
supplies to researchers for experiments generally has less than $4 \% \Delta^{9}-\mathrm{THC}$, and so findings from these experiments have limited implications for modern-day cannabis users.

\section{Cognition, learning and memory}

Endocannabinoids (eCBs) are, in a sense, the brain's own natural cannabis system, and $\Delta^{9}$ THC and other CB1R agonists alter brain levels of $\mathrm{eCBs}^{19,20}$. eCBs are neuroactive lipids that participate in a range of physiological processes including reward, motivation, emotional homeostasis, pain processing, and synaptic plasticity contributing to learning and memory. At present, the best-characterized eCBs are $\mathrm{N}$-arachidonylethanolamide (anandamide; AEA) and 2-arachidonoylglycerol (2-AG) $)^{21,22}$ and both of these lipids exert agonist activity at CB1Rs and CB2Rs. Owing to their lipid nature, AEA and 2-AG are not stored in vesicles but are synthesized on an 'on-demand' basis, and as such brain eCB levels are critically reliant on the balance between evoked biosynthesis and subsequent clearance by intracellular enzymemediated hydrolysis. eCBs are crucial in certain forms of neuronal plasticity, and $\Delta^{9}$-THC has been shown to disrupt long-term potentiation (a model for learning and memory) and longterm depression in preclinical studies ${ }^{23}$. In this section, we consider the acute and longer-term effects of cannabis on cognition, learning and memory, as well as effects potentially persisting after an individual has stopped using the drug. We also review evidence on the impact of starting cannabis use early in adolescence.

Acute effects. Acute effects are transient and seen in the time period during which the individual is intoxicated with the drug (e.g. feeling 'stoned' for around 5-120 minutes when smoked). A single dose of cannabis or its main active ingredient $\Delta^{9}$-THC robustly and dosedependently impairs working and episodic memory ${ }^{24,25}$. Memory impairments occur however the drug is administered, but the onset of effect is more rapid when it is inhaled or given 
intravenously than when it is ingested orally. Specifically, the encoding of new memories is impaired during cannabis intoxication and this leads to subsequent deficits in recalling these memories; by contrast, the retrieval of old memories that were consolidated when not under the influence is unaffected. Cannabis-induced working-memory deficits are seen more in the ability to manipulate information while it is 'online' (for example, when doing mental arithmetic) than in the ability to simply retain it for brief periods (for example, when remembering a telephone number before dialling it). Whereas on placebo, brain activity in the dorsolateral prefrontal cortex (DLPFC) increases linearly with a task's working memory load, acute dosing with $\Delta^{9}$-THC prevents this load-associated increase in DLPFC activity ${ }^{26}$.

These effects on memory are consistent with the extensive preclinical evidence of: the amnestic effects of cannabis in animal models; the high density of cannabinoid receptors in memoryassociated brain regions such as the hippocampus, amygdala and prefrontal cortex (Figure 1); and observations that $\Delta^{9}$-THC induces disruption of plasticity (including long-term potentiation (LTP) and long-term depression (LTD)) in the hippocampus and decreases acetylcholine release here and in the prefrontal cortex (PFC) (Figure 2A).

Some studies report acute $\Delta^{9}$-THC-induced impairment of behavioural inhibition and increases in impulsivity, but findings on attention, decision-making and risk-taking tasks are mixed and task-dependent ${ }^{25,27}$. There is also some evidence that acute effects may vary depending on an individual's previous level of use of the drug. Tolerance to the memory-impairing ${ }^{28}$ and psychomotor effects ${ }^{29}$ of acute $\Delta^{9}$-THC have been shown in individuals who use the cannabis more than once a week, probably reflecting a downregulation of cortical CB1Rs ${ }^{30,31}$ (Figure 2B).

There is some evidence that acute effects of $\Delta^{9}$-THC cannabis on memory depend on the particular type of cannabis ingested. Smoking cannabis with higher levels of CBD protected 
regular users against the acute memory-impairing effects of $\Delta^{9}-\mathrm{THC}^{10}$. Findings in cannabisusing volunteers replicated these protective effects of CBD on $\Delta^{9}$-THC-induced acute memory impairment ${ }^{11}$. Indeed, CBD alone has been shown to enhance fear extinction learning in humans ${ }^{7}$. This further supports the notion that CBD and $\Delta^{9}$-THC may have opposing effects on some of the neural substrates of human memory ${ }^{32}$. A recent cross-sectional study found that CBD appeared to protect against the effects of $\Delta^{9}$-THC on hippocampal volume loss ${ }^{33}$. Future research should determine whether chronic exposure to CBD might be protective in the longer term.

Long-term effects. Although in several countries legislation enables new studies of medical cannabis to use prospective, randomized controlled trial designs, to date studies of the neurocognitive effects of repeatedly using cannabis (that is, the long-term or chronic effects) have relied mainly on retrospective, self-reported drug use by people who choose to use cannabis recreationally, and, in most cases, illicitly. More objective indices of drug use can be obtained through hair samples, although such analysis has limitations (for example, they are influenced by hair dyes $)^{34}$ and have been rarely used in studies. Long-term impairments in memory have been reported mainly in frequent, heavy users, but confounding factors make it difficult to establish cause-effect relationships between cannabis use and changes in neurocognitive function. Such factors include baseline cognitive function prior to drug use; use of other cognitively impairing drugs like alcohol; types of cannabis used; age at which use started; and mental health problems, including depression and cannabis addiction.

Case-control studies of non-acute effects of cannabis have produced inconsistent findings to date, but tend to mirror acute findings. The most consistently reported long-term effects in these studies are impairments of encoding new episodic memories, with some studies finding 
persistent deficits in the first few days of abstinence but little evidence of persisting deficits at 28 days after use ${ }^{25}$. Meanwhile, the findings for measures of working memory, attention and impulsivity are mixed. One study ${ }^{35}$ found no difference in decision making or risk taking between cannabis users and non-users who were matched for potential mental health confounds. However, poorer decision-making accuracy in users was significantly associated with the number of symptoms of cannabis addiction.

Functional neuroimaging studies often reveal subtle differences between chronic cannabis users and controls in brain activity during performance of cognitive tasks; again, the persistence and clinical importance of these differences remain to be determined. A systematic review ${ }^{36}$ of 43 studies concluded that chronic cannabis use may alter brain structure and function in both adult and adolescent users (particularly in the medial temporal and frontal cortices, and cerebellum). However, the findings of the mainly cross-sectional studies displayed remarkable heterogeneity and it was not possible to infer causation. Further, deficits are most consistently observed only among heavy users — those most likely to be addicted to cannabis. Cognitive changes related to chronic exposure to cannabis or $\Delta^{9}$-THC observed in humans and in animal model studies (such as reduced inhibitory control and impaired decision making) $27,37,38$ are implicated in the maintenance of addiction ${ }^{39}$. in part by impairing the reversal of behaviors related to drug acquisition/taking that propel continued drug use despite negative consequences. It could thus be proposed that these cognitive changes may interact with genetic vulnerability factors to increase the risk of developing cannabis addiction.

Age-dependent effects: adolescence and adulthood. The $\mathrm{eCB}$ ) system has a major role in neurodevelopmental and maturational processes including synaptic pruning and white-matter development, and these processes are especially prevalent during adolescence. As exogenous cannabinoids affect the functioning of the eCB system, it is plausible that prolonged use during adolescence disrupts the neurodevelopmental maturational processes during this 
period $^{40}$. Thus, the human brain may be more vulnerable to drugs at the time when use of cannabis often begins.

Preclinical studies have shown that repeated exposure to $\Delta^{9}$-THC has a greater negative impact on the working memory, object recognition, and pre-pulse inhibition of adolescent than on adult rodents ${ }^{40}$. Chronic administration of CB1R agonists or $\Delta^{9}$-THC to adolescent rats produces persisting impairments in object recognition memory that are not seen with the same treatment in adult rats ${ }^{41,42}$, as well as greater alterations in the level of expression of various hippocampal proteins (which may account for adolescent-specific memory effects ${ }^{42}$ ). A single dose of $\Delta^{9}$-THC has also been found to result in greater acute impairments to spatial and nonspatial learning in adolescent rats than in adult rats ${ }^{43}$.

The age-related effects of cannabis use on cognitive function may therefore be dependent on the maturational state of the neural circuits that are affected by the drug. This may reflect the fact that $\Delta^{9}$-THC induces perturbations in the crucial influence of the eCB signalling that is involved in brain development, for processes including neural proliferation, morphogenesis, neural migration and synaptogenesis ${ }^{44-46}$. Consistent with this interpretation were the results of a study in which $\Delta^{9}$-THC was repeatedly administered over 6 months to adolescent monkeys in doses that corresponded well to human self-administration (approximately 1-2 joints, 5 days per week). This repeated administration blunted the usual pattern of accuracy improvements on a test of spatial working memory (which matures after object working memory), but not on an object working memory task ${ }^{47}$. Thus, the persistent effects of $\Delta^{9}$ THC on cognition in animals are more evident when exposure coincides with the developmental stage during which cannabinoid-affected neural circuits are actively maturing. 
Similarly, there is also accumulating evidence in humans that neurocognitive function and aspects of brain architecture are more disrupted by cannabis when individuals start using it during adolescence, although there is a scarcity of direct comparisons with adult users. Some structural imaging studies in adolescent and young adult cannabis users have reported decreased volume in several cortical and sub-cortical regions ${ }^{36}$ but findings across different studies vary considerably ${ }^{48}$. For example, although structural differences between adolescent cannabis users and controls in orbitofrontal cortex (OFC) volume have been found, smaller volumes at 12 years of age were shown to predict cannabis use at 16 , suggesting that differences in the OFC may be a vulnerability factor for use rather than a consequence ${ }^{49}$. And although smaller hippocampal volumes in cannabis users have been associated with age of onset of use, this association appears less consistent does the association between reductions in the size of the hippocampus and the amount of use, suggesting that the structure of the hippocampus may be more affected by duration and intensity of exposure rather than by early use specifically ${ }^{50}$.

Diffusion tensor imaging (DTI) studies have found poorer white-matter integrity (indexed by both lower fractional anisotropy and higher mean diffusivity) in adolescents who use cannabis frequently compared with control non-users ${ }^{48}$. Further, reductions in those indices of white-matter integrity correlate with deficits in measures of neurocognitive performance.

Several functional MRI (fMRI) studies suggest that there is increased BOLD signal in taskrelated areas in young cannabis users compared with non-using controls ${ }^{48}$. For example, Jager and colleagues ${ }^{51}$ assessed 13-19-year-old boys who had used cannabis at least 200 times in their lives and compared them with non-using, age-matched controls. The cannabis users showed greater activation in prefrontal regions during a working memory task than did controls. Overall, most functional imaging findings suggest that adolescent cannabis users 
show increased recruitment of neural resources — potentially reflecting compensatory activity — in brain areas subserving task-related processing.

In terms of neurocognitive function, individuals who started using cannabis during adolescence have been reported to have greater deficits in visuospatial attention ${ }^{52}$, verbal fluency ${ }^{53}$ and inhibition $^{53}$ than do those who start in adulthood. Importantly, based on the Dunedin prospective cohort data, one study ${ }^{54}$ concluded that having cannabis addiction that started during adolescence and persisted into adulthood was associated with a decline of around 8 IQ points (Box 1). However, two recent, large-scale studies cast doubt on a causal explanation (Box 1).

One limitation of the studies to date assessing the effects of cannabis use on the adolescent brain is that they have focused on age (whereby onset of use before 15-17 years of age is considered 'early') rather than on adolescent pubertal markers or potential sensitive periods that may more accurately index stage of brain development ${ }^{55}$. Since the eCB system interacts with gonadal hormones, and girls typically begin puberty earlier and reach pubertal maturation earlier than boys, pubertal stage may influence findings on sex differences. The few studies looking at acute effects of cannabis or $\Delta^{9}$-THC have produced little in the way of age-dependent sex differences although chronic differences may exist ${ }^{25}$. Younger age of regular cannabis use onset predicted poorer memory in women but not men $^{56}$. Surprisingly, the same study found earlier age of cannabis use initiation predicted better decision-making performance in both male and female cannabis users. Maturation of the PFC and its connections with the limbic system occurs earlier in girls ${ }^{57}$ and this difference may contribute to reported sex differences in the effects of adolescent cannabis use ${ }^{25,56}$. The paucity of studies of sex differences in the effects of cannabis limits conclusions and should be addressed by future research. 
Effects persisting after stopping use. Several studies of long-term effects after an individual stops using cannabis are converging to show that cognitive impairments do not persist beyond 4-6 weeks after abstinence ${ }^{58,59}$. Using positron emission tomography (PET) imaging, one study ${ }^{30}$ demonstrated that chronic cannabis users showed a downregulation of cortical CB1Rs that correlated with years of use. After $\sim 4$ weeks of continuously monitored abstinence from cannabis at a secure research unit, their CB1R density returned to control levels, further supporting recovery within 4 weeks, and even, according one recent study, after as little as 2 days $^{31}$. Reversible downregulation of brain CB1Rs after chronic exposure to cannabis has also been shown in rodent studies ${ }^{60}$. Other studies of 'persisting' effects have used structural and/or functional imaging but with cross-sectional designs, different abstention intervals and a range of confounds (including group differences in comorbid alcohol use and pre-cannabis level of functioning), which make it difficult to draw any causal conclusions. Longitudinal studies are thus needed to determine whether these effects of abstinence are seen even in those starting use in adolescence ${ }^{61}$.

\section{Cannabis addiction}

Much research on cannabis and mental health has focused on psychosis (Box 2), although addiction is a far more common problem: we estimate that people who try cannabis are 9-fold more likely to become addicted to it than to develop psychosis in their lifetime ${ }^{62-64}$. In this section, we introduce the concepts of cannabis addiction and withdrawal, review the rewarding effects of cannabinoids in relation to $\mathrm{eCB}$, dopaminergic, opioid and noradrenergic neurotransmitter systems, and highlight vulnerability factors and possible treatments for cannabis addiction. 
Cannabis addiction and withdrawal. The term 'addiction' is in a terminological quagmire; here, however, we define it as an acquired, chronic, relapsing disorder that is characterized by a powerful motivation to continually engage in an activity despite persistent negative consequences. Addictive drugs can all cause similar changes to brain circuits underpinning reward, salience, impulsivity, compulsivity, learning and memory ${ }^{39,65-67}$ although these differ according to class of drug (including cannabis) ${ }^{68,69}$. Clinical problems associated with cannabis use were previously diagnosed as 'cannabis abuse' or 'cannabis dependence' in the Diagnostic and Statistical Manual for Mental Disorders, fourth edition, text revision (DSM-IV-TR). In the most recent version (DSM-5) these categories were amalgamated into a single diagnosis of 'cannabis use disorder' (CUD) as described in Box 3. The chances of becoming addicted to cannabis after lifetime exposure is $8.9 \%$, which is comparatively lower than for cocaine (20.9\%), alcohol $(22.7 \%)$ or tobacco $(67.5 \%)^{64}$. Nevertheless, the clinical need for treatment of cannabis addiction is substantial and increasing in North America, Europe and Oceania ${ }^{5}$. Across Europe, cannabis now accounts for more first-time entrants to drug treatment services than does any other illicit drug ${ }^{70}$.

A specific cannabis withdrawal syndrome - one aspect of addiction - is well recognized and affects around $50 \%$ of daily users upon cessation of use, and typically begins 1-2 days after cessation, peaks at 2-6 days and remits at $1-2$ weeks $^{71}$. Prominent symptoms include craving, sleep problems, nightmares, anger, irritability, dysphoria and nausea ${ }^{72}$. Cannabis withdrawal symptoms correlate with reductions in CB1R availability during acute abstinence ${ }^{31}$ and can be alleviated by $\Delta^{9}$-THC in a dose-dependent manner ${ }^{73} . \Delta^{9}$-THC withdrawal is associated with increased release of the stress peptide corticotropin releasing-factor (CRF) in the central nucleus of the amygdala ${ }^{74,75}$. Similar increases in amygdalar CRF release are evident during withdrawal from most classes of recreational drugs (including nicotine, alcohol, 
psychostimulants and opiates), and contribute to negative affective states and decreased brain reward function ${ }^{76}$. It is therefore noteworthy that cannabis is frequently rolled with tobacco in ‘joints' and many users also smoke cigarettes (Box 4). In daily users of cannabis and tobacco, individual withdrawal effects appear similar for both drugs; combined withdrawal produces stronger effects than does withdrawal for either one alone ${ }^{77}$.

Cannabinoids and reward. $\Delta^{9}$-THC produces the effects cannabis users seek; they report liking it and wanting more ${ }^{24}$. Additionally, cannabis with higher $\Delta^{9}$-THC content (for example, $3.5 \%$ versus $2.0 \%$ ) produces stronger reinforcement in human choice paradigms ${ }^{78}$. Since reinforcement of drug use is considered to be one component in the transition from voluntary to compulsive use ${ }^{67}$, these findings suggest that high-THC cannabis might increase vulnerability to addiction. However, it is difficult to extrapolate these findings to modern, highpotency cannabis with $\sim 15 \% \Delta^{9}-\mathrm{THC}^{16-18}$. Recent naturalistic studies indicate that people adapt to rising $\Delta^{9}$-THC concentrations by adding less cannabis to their joints ${ }^{79}$ and/or inhaling less smoke ${ }^{80}$. Nevertheless, cross-sectional data suggest that use of high-THC forms of cannabis is associated with greater addiction severity ${ }^{81}$. It is therefore possible that recent increases in cannabis potency ${ }^{15}, 16$ might have contributed to the rising demand for treatment of cannabis $\operatorname{addiction}^{5,70}$.

The presence or absence of CBD in evaluations of $\Delta^{9}$-THC reward may also be relevant. One study found that people who smoked cannabis containing only low levels of CBD were more prone to have their attention captured by cannabis-related stimuli than were those smoking high-CBD cannabis ${ }^{82}$. This suggests that CBD could protect against addiction, as attentional bias toward drug-related stimuli correlates with craving and is sensitive to relapse-provoking 
manipulations ${ }^{83}$. However, CBD does not influence the acute reinforcing effects of cannabis or the rewarding feeling of being 'stoned' $82,84,85$.

The rewarding effects of cannabinoids in animals depend on species, route of administration and experimental design. Rats will perform an operant behaviour to receive $\Delta^{9}$-THC infusions into the brain ventricular space and to receive CB1R agonist infusions into the nucleus accumbens shell and posterior $\mathrm{VTA}^{86,87}$, as with other drugs of abuse. However, there has been substantial difficulty in establishing operant intravenous $\Delta^{9}$-THC self-administration in rodents $^{78}$, possibly owing to the prolonged pharmacokinetic effects of $\Delta^{9}$-THC that impede establishment of discrete response-reward associations. The higher cognitive function in primates may allow for clearer discernment as to whether lever pressing behavior is causal for the somewhat delayed alterations in reward state resulting from intravenous $\Delta^{9}$-THC. It is also possible that the aversive and motor-depressant effects of $\Delta^{9}$-THC present greater impediments to self-administration in rodents than in primates.

Consistent with the human literature, studies in rats also demonstrate that $\Delta^{9}$-THC reward is dose-dependent. These effects appear to follow an inverted U-shaped curve whereby high $\Delta^{9}-$ THC doses are less reinforcing than medium doses. For example, as indexed using the intracranial self-stimulation (ICSS) paradigm, reward system function in the rodent brain is enhanced by low doses of $\Delta^{9}-\mathrm{THC}^{88}$, whereas higher doses and more-potent CB1R agonists can decrease this function ${ }^{89,90}$. Consistent with this, the rewarding effects of low doses of $\Delta^{9}$ THC or CB1R agonists in the conditioned place-preference paradigm are supplanted by aversive effects at higher doses ${ }^{86,91,92}$. An inverted-U shaped profile is consistent with data from human studies that indicate that high-THC cannabis is preferred and associated with greater addiction severity than low-THC cannabis ${ }^{81}$ whereas extremely potent products (such 
as synthetic CB1R agonists) are less addictive than 'natural' cannabis, have more negative effects and are only preferred by $7 \%$ of users ${ }^{93}$.

In animals, CB1R antagonism produces a 'rightward' shift along this inverse U-shaped rewardaversion dose-effect function, blocking the rewarding effects of low doses of CB1R agonists and preventing the aversive effects of high CB1R agonist doses. This underscores the bimodal effects of CB1R activity on brain reward processing. Squirrel monkeys voluntarily selfadminister intravenous $\Delta^{9}-$ THC $^{94}$ and rats reliably self-administer synthetic CB1R agonists ${ }^{95}$, 96 - actions that are prevented by CB1R antagonism. Taken together, these studies suggest that the CB1R activity has a crucial role in cannabinoid reinforcement and cannabis addiction.

Similar to $\Delta_{9}-\mathrm{THC}$ and many synthetic cannabinoid agonists, eCBs exhibit agonist effects at brain CB1Rs. The eCB AEA takes its name from the Sanskrit word 'ananda', meaning 'bliss'. Although pharmacologically enhanced eCB signalling (for example, through the inhibition of eCB clearance) generally does not produce rewarding effects per se, persistent disruptions in eCB signalling appear to contribute to facets of drug dependence across drug classes ${ }^{97,} 98$. CB1R downregulation in chronic cannabis users has been reported in three studies ${ }^{30,31,99}$; these effects subside within days to several weeks of sustained abstinence ${ }^{30}{ }^{31}$. Similarly, rodents chronically exposed to $\Delta^{9}-\mathrm{THC}$ or synthetic CB1R agonists exhibit a reduction in CB1R function throughout the brain ${ }^{100,101}$ that persists for days to weeks following $\Delta^{9}$-THC treatment, followed by a functional recovery that varies between brain regions ${ }^{102}$. eCBmediated forms of synaptic plasticity in the nucleus accumbens and hippocampus are abolished following exposure to $\Delta^{9}$-THC or CB1R agonists ${ }^{101,103,104}$ — and this may substantially impact reward processing and memory processes mediated by these regions. Chronic exposure to $\Delta^{9}$ THC or CB1R agonists increases enzymatic clearance of AEA and reduces brain tissue AEA 
content in rodents ${ }^{19,105,106}$, and consistent with these data, frequent cannabis smokers exhibit decreased AEA levels in CSF $^{107}$. Although evidence is limited, elevated serum AEA levels may be evident in ex-users of the drug ${ }^{108}$.

Dopamine, opioids and noradrenaline. Human PET studies indicate that $\Delta^{9}$-THC can increase dopamine release in the striatum ${ }^{109}$ although to a far lesser extent than do other recreational drugs, and not in all studies ${ }^{110}$. In rodents, $\Delta^{9}$-THC and CB1R agonists increase the firing rate and bursting activity of VTA dopamine neurons, resulting in dose-dependent increases in the mesocorticolimbic release of dopamine ${ }^{111-113}$. Conversely, withdrawal from chronic $\Delta^{9}$-THC or CB1R agonist exposure is associated with decreased firing of dopaminergic cells and decreased dopamine release in the nucleus accumbens ${ }^{114,}{ }^{115}$. Consistent with these observations in animal models, reduced capacity to synthesize striatal dopamine was recently reported in human cannabis users, particularly among addicted individuals ${ }^{116}$. However, in humans, chronic cannabis exposure is not typically associated with abnormalities in striatal dopamine release or in $\mathrm{D}_{2}$ receptor expression ${ }^{117}$ and, together with the modest dopaminergic effects of acute $\Delta^{9}$-THC, the available human data provide only weak support for dopaminergic involvement in cannabis addiction.

$\Delta^{9}$-THC-induced increases in opioid peptide release ${ }^{118,119}$ may also contribute to the rewarding effects of cannabis. The opioid receptor antagonist naltrexone reduces $\Delta^{9}$-THC-induced increases in mesolimbic dopamine release, intravenous $\Delta^{9}$-THC self-administration and intracerebroventricular CB1R agonist self-administration in rats and monkeys ${ }^{120,121}$. Sixteen days of naltrexone treatment reduced self-administration and some positive subjective effects of $\Delta^{9}$-THC in humans ${ }^{122}$. 
$\Delta^{9}$-THC and other CB1R agonists stimulate noradrenergic cell firing and increase levels of noradrenaline in multiple brain regions in which the neurotransmitter can impact motivated behaviours $^{123}$ — including the nucleus accumbens, prefrontal and cerebral cortices, hippocampus and hypothalamus ${ }^{124-126}$. The behavioural importance of CB1R agonist-induced increases in noradrenaline release has not been carefully evaluated, although evidence suggests it may contribute to the aversive (but not anxiogenic) effects of high doses of CB1R agonists ${ }^{127}$. For example, the aversive effects of high doses of the CB1R agonist WIN 55,212-2 (as measured in the conditioned place-aversion paradigm) are reduced by attenuation of noradrenergic signalling in the nucleus accumbens, although WIN 55,212-2-induced anxietylike behaviour (as measured on the elevated plus-maze) is not reduced by these manipulations $^{127}$.

Vulnerability factors. Irrespective of the drug constituents ${ }^{81,82}$, only a minority of cannabis users become addicted; therefore, other factors must predict vulnerability. Concurrent tobacco use has been identified as a risk factor in a number of studies ${ }^{128-130}$, as have early adolescent onset and frequent (especially daily) use ${ }^{131} 128$. Males typically have an earlier opportunity to use cannabis, a greater risk of addiction and a faster progression from first opportunity of use to addiction ${ }^{64,128,129}$. These findings are consistent with normative data from European treatment services: the mean age of first treatment is 24 , the mean age at first cannabis use is 16 and $83 \%$ of treated individuals are male ${ }^{70}$.

Interestingly, a 3-year prospective study of daily users found that variables related directly to cannabis use did not predict transition to addiction; more important were current factors such as living alone, coping motives and negative life events (such as having had a major financial crisis $)^{80}$. A meta-analysis of 24 twin studies ${ }^{132}$ suggested that genetic influences account for 
$55 \%$ of the vulnerability to cannabis addiction, with shared environmental factors and nonshared environmental factors accounting for much lower proportions $(17.5 \%$ and $27.5 \%$, respectively). Although isolated studies have identified specific gene variants associated with increased risk of developing cannabis use disorder (reviewed in ref. ${ }^{133}$ ), cannabis addiction phenotypes are likely to be polygenic, and genotypes probably overlap with those linked to substance addiction in general $^{134}$.

Possible treatments for cannabis addiction. Cannabis addiction is not easily treated by psychological therapies ${ }^{135}$, and although many pharmacotherapies, have been tested ${ }^{136}$ including antidepressants, anxiolytics, noradrenaline-reuptake inhibitors, anticonvulsants, glutamatergic modulators and CB1R agonists — none has been approved. Based on existing clinical trial data, a 12-week trial of the GABA mimetic gabapentin ${ }^{137}$ and an 8-week trial of $N$-acetylcysteine ${ }^{138}$ have shown promise for reducing cannabis use according to urine sampling during treatment. Gabapentin also improved a number of secondary outcomes, including withdrawal symptoms, executive function and self-reported depression ${ }^{137}$. CB1R agonists such as dronabinol (oral synthetic $\Delta^{9}-$ THC) ${ }^{139}$ and nabiximols ${ }^{140}$ (an oral spray containing $\Delta^{9}$-THC and CBD in equal ratio) attenuated cannabis withdrawal symptoms and improved treatment retention, but did not reduce cannabis use compared with placebo. These findings suggest that substitution treatments can replace $\Delta^{9}$-THC in cannabis but are not sufficient to promote abstinence from it.

Few studies have evaluated the contribution of dysregulated eCB signalling to cannabis addiction and related physiological and behavioural disruptions. However, eCBs provide important homeostatic regulation over emotional state ${ }^{141}$ and sleep function ${ }^{142}$, and so it is plausible that $\Delta^{9}$-THC-induced impairment of eCB signalling contributes to the negative 
emotional states and sleep disturbances that are present during protracted cannabis abstinence ${ }^{71-73}$. Intriguingly, studies in rodents demonstrate a palliative effect of 2-AGclearance inhibitors on the somatic symptoms of CB1R-antagonist-precipitated $\Delta^{9}$-THC withdrawal ${ }^{105}$. Collectively, these observations have led to the proposed use of eCB-clearance inhibitors as treatments for cannabis withdrawal, and perhaps addiction ${ }^{143,144}$. Given the largely unmet clinical need, developing effective pharmacological treatments should be a top research priority.

\section{Cannabis, anxiety and depression}

Like most addictions, cannabis addiction is often comorbid with other mental health problems. Epidemiological evidence indicates a possible association between regular cannabis use and the development of anxiety and depression. However, the evidence is more mixed and less consistent than that between cannabis use and psychosis ${ }^{62}$. One recent study compared the mental health of individuals who were addicted to cannabis (according to the DSM-IV) with that of non-addicted cannabis users who had similar patterns of cannabis use. Only the addicted users had depression and anxiety problems ${ }^{145}$. Compared with general population norms, nonaddicted frequent users were more likely to show externalizing disorders (such as attentiondeficit hyperactivity disorder) that were likely to have predated their cannabis use. Otherwise, these individuals were similar in terms of mental health to the general population, suggesting that cannabis contributes to mental health problems only in those who are vulnerable for other reasons.

Depression and anxiety disorders not only are associated with cannabis addiction ${ }^{146}$ but also are predictive of whether individuals transition from use to addiction ${ }^{147}$. Strikingly, a high number of cases of depression and anxiety disorders were reported among obese individuals who were treated with the anti-obesity drug Rimonabant, a CB1R antagonist. Many of these 
individuals had no prior history of these disorders ${ }^{148,} 149$ and this led to the withdrawal of Rimonabant from therapeutic use. These findings suggest that CB1R antagonists increase the risk of depression and/or anxiety. Moreover, preclinical studies have shown that mice that genetically lack CB1Rs show increased depressive-like symptoms ${ }^{150}$ and, in wild-type mice, CBD has antidepressant effects ${ }^{151}$. Rodent studies have implicated the eCB system in the regulation of emotion ${ }^{152}$. Similarly, there are also data from rodent studies suggesting that impaired CB1R signalling leads to depression-like symptoms, and that enhancement of CB1R signalling produces antidepressant-like behavioural effects in rodents ${ }^{153}$.

In our own studies of young (16-24-year-old) daily cannabis users, we have found that levels of $\Delta^{9}$-THC in hair are significantly associated with self-reported levels of both depression and anxiety ${ }^{154}$. However, a recent epidemiological study ${ }^{146}$ suggested that increases in self-reported depression in cannabis users are not long-lasting, as no consistent associations were found between adolescent cannabis use and depression at age 29. By contrast, the same study showed daily cannabis use and cannabis addiction in early adulthood was associated with more than double the non-user control rate of anxiety disorders at 29. The association between cannabis use and anxiety may arise because the same factors that predispose people to use cannabis also predispose them to anxiety. Indeed, there is accumulating evidence that in vulnerable individuals, cannabis is often 'used' to self-medicate social anxiety ${ }^{155,156}$. This is interesting, as controlled studies of acute effects in humans have shown that $\Delta^{9}$-THC increases anxiety ${ }^{6}$, whereas CBD decreases it ${ }^{9}$. Furthermore, $\Delta^{9}$-THC and CBD acutely produce opposite but subtle effects on human facial affect recognition ${ }^{84}$ and amygdala activation when viewing fearful faces ${ }^{32}$. 
The interconnectedness of cannabis use, mental health problems and cognitive functioning is important. It is inherently difficult to determine causality in the type of studies discussed above because factors besides cannabis use (e.g. premorbid cognitive and emotional function) may be directly associated with risk of mental illness. Such factors could predispose an individual both to mental illness and to using cannabis ${ }^{3}$, and the combination of these disorders would in turn increase their impact upon cognitive functioning.

\section{Conclusions and future directions}

Cannabis has been used for thousands of years for a range of medicinal properties as well as for its desired psychological and social effects, which recreational users value. This use can, however, carry a penalty: a range of undesired effects that vary in the severity of their impact on the individual's life. Although evidence of clear causality is lacking, these undesired effects may range from mild cognitive impairment to disabling psychiatric disorders. However, most recreational and medical users appear to rate the benefits as outweighing the risks in choosing to continue their cannabis use. It should be noted that public health messages to users are distorted, because funding for research is often targeted to studying the harmful effects of cannabis and ignores benefits. Despite studies aiming to document negative effects, occasionally positive effects are noted such as enhanced divergent thinking following either oral THC or smoked cannabis ${ }^{24,157}$. Future research should evaluate perceived benefits to give a more balanced understanding; people clearly do not use cannabis only for its harms.

Throughout this article we have specified gaps in our knowledge. Although problems associated with cannabis use are mainly observed in heavy, frequent users, we are still not sure 
what level of use of what type of cannabis is non-problematic. We need such data for harmreduction advice for both medical and recreational users.

Another question that needs answering is: how does repeated use of cannabis causally affect the human adolescent and adult brain given that most cannabis users also use alcohol, another cognitively impairing drug? A recent comparison of daily cannabis users who also drank alcohol and alcohol-intake-matched, non-using controls in both adolescents and adults found no differences in brain structure between the two groups ${ }^{158}$, but longitudinal data are currently lacking. It is also still unclear exactly how the effects of cannabis vary across clinical populations. For example, a meta-analysis of showed that people who are diagnosed with schizophrenia and use cannabis function better cognitively than individuals with schizophrenia who do not use cannabis ${ }^{159}$. Finally, we need to understand more about how variants of the drug produce differential effects — variants not only in constituent cannabinoids but also the new synthetic cannabis variants (such as 'spice') ${ }^{93}$ as well as new forms of administration (for example, through vaping or edible forms) $)^{160}$.

Future studies will help to fill these gaps of knowledge, especially if they incorporate methodological improvements. Most studies of long-term effects assess level of cannabis use through various self-report measures (such as frequency, years of use, time to smoke a specific amount of the drug) and could benefit from improved biomarkers such as $\Delta^{9}$-THC and CBD levels in hair. We need longitudinal studies that follow young people from before puberty (that is, prior to drug use), through biologically defined puberty and into adulthood (while monitoring drug use) and again after subsequent abstinence or not. Such studies would use a comprehensive range of assessments (including: tests of cognition, motivation, brain function and mental health; biomarkers of different types; and quantities of cannabis or cannabinoids 
and other recreational substances used) so the interactions among these factors may be monitored. This need should be addressed by the US National Institutes of Health-funded Adolescent Brain Cognition Development (ABCD) Study: a prospective, 10-year, longitudinal study of 10,000 individuals beginning at 9-10 years of age. This study is designed to assess the impact of substance use on brain development and neurocognitive function through a battery of measures obtained prior to cannabis use, following use at various levels and again following cessation of use. Given recent changes in the medical and legal status of cannabis in some countries, randomized controlled trials of the effects of different types and doses of cannabis can now be conducted. Work with animals will be important in delineating chronic effects, and studies administering doses and schedules within a human-relevant range (as exemplified in Ref. ${ }^{47}$ ) are the most helpful.

The number of cannabis addicts may well grow as cannabis use becomes more acceptable and the drug more accessible. Indeed, there is evidence that this is already happening in the United States ${ }^{161}$. It is therefore urgent that we increase efforts to understand what factors influence the development of addiction and build preventative efforts against this. Currently, we lack an effective pharmacological treatment for cannabis addiction that can be used conjointly with psychosocial therapies to boost the current low efficacy of all treatment approaches. This need requires urgent research attention.

With hindsight, we can clearly see the enormous problems that have been caused to many individuals and to society by tobacco and alcohol. Unlike cannabis, these drugs are legal in most countries, despite the fact that, if asked to decide today which psychoactive drugs should be legal, cannabis (which rarely kills people) might well be judged as being comparatively benign. Legislative changes would help researchers, as current restrictive drug scheduling 
markedly hinders neuroscience research and the innovation of psychiatric treatment ${ }^{162}$. More importantly, if handled carefully from a harm-reduction standpoint, a regulated market might increase the control over the age of initiation of use and other vulnerability factors; inform accurately about dosage; and increase the availability of more-balanced cannabis (that is, with lower levels of $\Delta^{9}$-THC and higher levels of CBD) to maintain desired effects while reducing the incidence of harms.

\section{References}

1. Whiting, P.F. et al. Cannabinoids for medical use: a systematic review and meta-analysis. Jama 313, 2456-2473 (2015).

2. Curran, H.V. \& Morgan, C.J.A. Desired and Undesired Effects of Cannabis on the Human Mind and Psychological Well-Being (ed. Hall, W.) (Oxford, United Kingdom, 2014).

3. Volkow, N.D., Baler, R.D., Compton, W.M. \& Weiss, S.R. Adverse health effects of marijuana use. New England Journal of Medicine 370, 2219-2227 (2014).

4. Hall, W. What has research over the past two decades revealed about the adverse health effects of recreational cannabis use? Addiction 110, 19-35 (2015).

5. UNODC. United Nations Office on Drugs and Crime. World Drug Report (2015).

6. D'Souza, D.C. et al. The psychotomimetic effects of intravenous delta-9-tetrahydrocannabinol in healthy individuals: implications for psychosis. Neuropsychopharmacology 29, 1558-1572 (2004).

7. Das, R.K. et al. Cannabidiol enhances consolidation of explicit fear extinction in humans. Psychopharmacology 226, 781-792 (2013).

8. Leweke, F. et al. Cannabidiol enhances anandamide signaling and alleviates psychotic symptoms of schizophrenia. Translational psychiatry 2, e94 (2012).

9. Bergamaschi, M.M. et al. Cannabidiol reduces the anxiety induced by simulated public speaking in treatment-naive social phobia patients. Neuropsychopharmacology 36, 1219-1226 (2011).

10. Morgan, C.J., Schafer, G., Freeman, T.P. \& Curran, H.V. Impact of cannabidiol on the acute memory and psychotomimetic effects of smoked cannabis: naturalistic study. The British Journal of Psychiatry 197, 285-290 (2010).

11. Englund, A. et al. Cannabidiol inhibits THC-elicited paranoid symptoms and hippocampal-dependent memory impairment. Journal of Psychopharmacology 27, 19-27 (2013).

12. Pertwee, R. The diverse CB1 and CB2 receptor pharmacology of three plant cannabinoids: $\Delta 9$ tetrahydrocannabinol, cannabidiol and $\Delta$ 9-tetrahydrocannabivarin. British journal of pharmacology 153, 199-215 (2008).

13. Muniyappa, R. et al. Metabolic effects of chronic cannabis smoking. Diabetes Care 36, 2415-2422 (2013).

14. Batalla, A. et al. Neuroimaging studies of acute effects of THC and CBD in humans and animals: a systematic review. Current pharmaceutical design 20, 2168-2185 (2014).

15. ElSohly, M.A. et al. Changes in cannabis potency over the last two decades (1995-2014)-Analysis of current data in the united states. Biological Psychiatry doi:10.1016/j.biopsych.2016.01.004 (2016).

16. Hardwick, S. \& King, L.A. Home Office cannabis potency study 2008 (Home Office Scientific Development Branch United Kingdom, 2008).

17. Niesink, R.J., Rigter, S., Koeter, M.W. \& Brunt, T.M. Potency trends of $\Delta 9$-tetrahydrocannabinol, cannabidiol and cannabinol in cannabis in the Netherlands: 2005-15. Addiction (2015).

18. Swift, W., Wong, A., Li, K.M., Arnold, J.C. \& McGregor, I.S. Analysis of cannabis seizures in NSW, Australia: cannabis potency and cannabinoid profile. PloS one 8, e70052 (2013). 
19. Di Marzo, V. et al. Enhancement of anandamide formation in the limbic forebrain and reduction of endocannabinoid contents in the striatum of delta9-tetrahydrocannabinol-tolerant rats. J Neurochem 74, 1627-35 (2000).

20. Gonzalez, S. et al. Behavioral and molecular changes elicited by acute administration of SR141716 to Delta9-tetrahydrocannabinol-tolerant rats: an experimental model of cannabinoid abstinence. Drug Alcohol Depend 74, 159-70 (2004).

21. Hillard, C.J. Chapter One-The Endocannabinoid Signaling System in the CNS: A Primer. International review of neurobiology 125, 1-47 (2015).

22. Mechoulam, R., Hanuš, L.O., Pertwee, R. \& Howlett, A.C. Early phytocannabinoid chemistry to endocannabinoids and beyond. Nature Reviews Neuroscience (2014).

23. Zhu, P.J. Endocannabinoid signaling and synaptic plasticity in the brain. Critical Reviews ${ }^{\mathrm{TM}}$ in Neurobiology 18 (2006).

24. Curran, H.V., Brignell, C., Fletcher, S., Middleton, P. \& Henry, J. Cognitive and subjective doseresponse effects of acute oral $\Delta 9$-tetrahydrocannabinol (THC) in infrequent cannabis users.

Psychopharmacology 164, 61-70 (2002).

25. Crane, N.A., Schuster, R.M., Fusar-Poli, P. \& Gonzalez, R. Effects of cannabis on neurocognitive functioning: recent advances, neurodevelopmental influences, and sex differences. Neuropsychology review 23, 117-137 (2013).

26. Bossong, M.G. et al. Effects of $\delta 9$-tetrahydrocannabinol on human working memory function. Biological psychiatry 71, 693-699 (2012).

27. Crean, R.D., Crane, N.A. \& Mason, B.J. An evidence based review of acute and long-term effects of cannabis use on executive cognitive functions. Journal of addiction medicine 5, 1 (2011).

28. D'Souza, D.C. et al. Blunted psychotomimetic and amnestic effects of $\Delta-9$-tetrahydrocannabinol in frequent users of cannabis. Neuropsychopharmacology 33, 2505-2516 (2008).

29. Ramaekers, J.G. et al. Tolerance and cross-tolerance to neurocognitive effects of THC and alcohol in heavy cannabis users. Psychopharmacology 214, 391-401 (2011).

30. Hirvonen, J. et al. Reversible and regionally selective downregulation of brain cannabinoid CB1 receptors in chronic daily cannabis smokers. Molecular psychiatry 17, 642-649 (2012).

This was the first study to report downregulation of CB1Rs in frequent cannabis users: the effects werereversed after 4 weeks of monitored abstinence, consistent with findings in rodents.

31. D'Souza, D.C. et al. Rapid changes in CB1 receptor availability in cannabis dependent males after abstinence from cannabis. Biological Psychiatry: Cognitive Neuroscience and Neuroimaging (2015). This paper reported a reversal of the downregulation of CB1Rs in frequent cannabis users after only 2 days.

32. Bhattacharyya, $\mathrm{S}$. et al. Opposite effects of $\Delta$-9-tetrahydrocannabinol and cannabidiol on human brain function and psychopathology. Neuropsychopharmacology 35, 764-774 (2010).

33. Yücel, M. et al. Hippocampal harms, protection and recovery following regular cannabis use.

Translational psychiatry (2016).

This cross-sectional study used hair analysis to examine the relationship between cannabinoids and hippocampal integrity and found that chronic $\Delta^{9}$-THC exposure is associated with reduced hippocampal volume and $\mathrm{N}$-acetylaspartate concentrations; however, these effects are not found in those with CBD as well as $\Delta^{\mathbf{9}}$-THC in hair or after extended abstinence from the drug.

34. Han, E., Chung, H. \& Song, J.M. Segmental hair analysis for 11-Nor- $\Delta$ 9-tetrahydrocannabinol-9carboxylic acid and the patterns of cannabis use. Journal of analytical toxicology 36, 195-200 (2012).

35. Gonzalez, R. et al. Performance of young adult cannabis users on neurocognitive measures of impulsive behavior and their relationship to symptoms of cannabis use disorders. Journal of clinical and experimental neuropsychology 34, 962-976 (2012).

36. Batalla, A. et al. Structural and functional imaging studies in chronic cannabis users: a systematic review of adolescent and adult findings. PloS one 8, e55821 (2013).

37. Irimia, C., Polis, I.Y., Stouffer, D. \& Parsons, L.H. Persistent effects of chronic Delta9-THC exposure on motor impulsivity in rats. Psychopharmacology (Berl) (2015).

38. Kucewicz, M.T., Tricklebank, M.D., Bogacz, R. \& Jones, M.W. Dysfunctional prefrontal cortical network activity and interactions following cannabinoid receptor activation. J Neurosci 31, 15560-8 (2011).

39. Goldstein, R.Z. \& Volkow, N.D. Dysfunction of the prefrontal cortex in addiction: neuroimaging findings and clinical implications. Nature Reviews Neuroscience 12, 652-669 (2011).

40. Lubman, D.I., Cheetham, A. \& Yücel, M. Cannabis and adolescent brain development. Pharmacology \& therapeutics 148, 1-16 (2015). 
41. Schneider, M. \& Koch, M. The effect of chronic peripubertal cannabinoid treatment on deficient object recognition memory in rats after neonatal mPFC lesion. European neuropsychopharmacology 17, 180186 (2007).

42. Quinn, H.R. et al. Adolescent rats find repeated $\triangle 9$-THC less aversive than adult rats but display greater residual cognitive deficits and changes in hippocampal protein expression following exposure. Neuropsychopharmacology 33, 1113-1126 (2008).

43. Cha, Y.M., White, A.M., Kuhn, C.M., Wilson, W.A. \& Swartzwelder, H. Differential effects of delta 9-THC on learning in adolescent and adult rats. Pharmacology Biochemistry and Behavior 83, 448-455 (2006).

44. Berghuis, P. et al. Endocannabinoids regulate interneuron migration and morphogenesis by transactivating the TrkB receptor. Proc Natl Acad Sci U S A 102, 19115-20 (2005).

45. Kim, D. \& Thayer, S.A. Cannabinoids inhibit the formation of new synapses between hippocampal neurons in culture. J Neurosci 21, RC146 (2001).

46. Mulder, J. et al. Endocannabinoid signaling controls pyramidal cell specification and long-range axon patterning. Proc Natl Acad Sci U S A 105, 8760-5 (2008).

47. Verrico, C.D., Gu, H., Peterson, M.L., Sampson, A.R. \& Lewis, D.A. Repeated $\Delta 9-$ tetrahydrocannabinol exposure in adolescent monkeys: persistent effects selective for spatial working memory. (2014).

This study provided proof-of-concept evidence in non-human primates that chronic exposure to exogenous cannbinoids during adolesence selectively disrupts cognitive processes that were actively developing during the time window of exposure.

48. Jacobus, J. \& Tapert, S.F. Effects of cannabis on the adolescent brain. Current pharmaceutical design 20, 2186 (2014).

49. Cheetham, A. et al. Orbitofrontal volumes in early adolescence predict initiation of cannabis use: a 4year longitudinal and prospective study. Biological psychiatry 71, 684-692 (2012).

50. Lorenzetti, V., Solowij, N., Fornito, A., Ian Lubman, D. \& Yucel, M. The association between regular cannabis exposure and alterations of human brain morphology: an updated review of the literature. Current pharmaceutical design 20, 2138-2167 (2014).

51. Jager, G., Block, R.I., Luijten, M. \& Ramsey, N.F. Cannabis use and memory brain function in adolescent boys: A cross-sectional multicenter functional magnetic resonance imaging study. Journal of the American Academy of Child \& Adolescent Psychiatry 49, 561-572. e3 (2010).

52. Ehrenreich, H. et al. Specific attentional dysfunction in adults following early start of cannabis use. Psychopharmacology 142, 295-301 (1999).

53. Gruber, S.A., Sagar, K.A., Dahlgren, M.K., Racine, M. \& Lukas, S.E. Age of onset of marijuana use and executive function. Psychology of Addictive Behaviors 26, 496 (2012).

54. Meier, M.H. et al. Persistent cannabis users show neuropsychological decline from childhood to midlife. Proceedings of the National Academy of Sciences 109, E2657-E2664 (2012).

55. Blakemore, S.J., Burnett, S. \& Dahl, R.E. The role of puberty in the developing adolescent brain. Human brain mapping 31, 926-933 (2010).

56. Crane, N.A., Schuster, R.M., Mermelstein, R.J. \& Gonzalez, R. Neuropsychological sex differences associated with age of initiated use among young adult cannabis users. Journal of clinical and experimental neuropsychology, 1-13 (2015).

57. Lenroot, R.K. \& Giedd, J.N. Sex differences in the adolescent brain. Brain and cognition 72, 46-55 (2010).

58. Pope, H.G., Gruber, A.J., Hudson, J.I., Huestis, M.A. \& Yurgelun-Todd, D. Neuropsychological performance in long-term cannabis users. Archives of General Psychiatry 58, 909-915 (2001).

59. Schreiner, A.M. \& Dunn, M.E. Residual effects of cannabis use on neurocognitive performance after prolonged abstinence: A meta-analysis. Experimental and clinical psychopharmacology 20, 420 (2012).

60. Sim-Selley, L.J. Regulation of cannabinoid CB1 receptors in the central nervous system by chronic cannabinoids. Critical Reviews ${ }^{\mathrm{TM}}$ in Neurobiology 15 (2003).

61. Fried, P., Watkinson, B. \& Gray, R. Neurocognitive consequences of marihuana-a comparison with pre-drug performance. Neurotoxicology and teratology 27, 231-239 (2005).

62. Moore, T.H. et al. Cannabis use and risk of psychotic or affective mental health outcomes: a systematic review. The Lancet 370, 319-328 (2007).

63. Kessler, R.C. et al. Lifetime and 12-month prevalence of DSM-III-R psychiatric disorders in the United States: results from the National Comorbidity Survey. Archives of general psychiatry 51, 8-19 (1994).

64. Lopez-Quintero, C. et al. Probability and predictors of transition from first use to dependence on nicotine, alcohol, cannabis, and cocaine: results of the National Epidemiologic Survey on Alcohol and Related Conditions (NESARC). Drug Alcohol Depend 115, 120-30 (2011). 
65. Koob, G.F. \& Volkow, N.D. Neurocircuitry of addiction. Neuropsychopharmacology 35, 217-38 (2010).

66. Nestler, E.J. Is there a common molecular pathway for addiction? Nat Neurosci 8, 1445-9 (2005).

67. Everitt, B. J., \& Robbins, T. W. (2016). Drug addiction: updating actions to habits to compulsions ten years on. Annual Review of Psychology, 67, 23-50.

68. Badiani, A., Belin, D., Epstein, D., Calu, D. \& Shaham, Y. Opiate versus psychostimulant addiction: the differences do matter. Nature Reviews Neuroscience 12, 685-700 (2011).

69. Fratta, W. \& Fattore, L. Molecular mechanisms of cannabinoid addiction. Current opinion in neurobiology 23, 487-492 (2013).

70. EMCDDA. European Drug Report. European Monitoring Centre for Drugs and Drug Addiction. (2015).

71. Budney, A.J., Hughes, J.R., Moore, B.A. \& Vandrey, R. Review of the validity and significance of cannabis withdrawal syndrome. American journal of Psychiatry 161, 1967-1977 (2004).

72. Allsop, D.J., Norberg, M.M., Copeland, J., Fu, S. \& Budney, A.J. The cannabis withdrawal scale development: patterns and predictors of cannabis withdrawal and distress. Drug and Alcohol dependence 119, 123-129 (2011).

73. Budney, A.J., Vandrey, R.G., Hughes, J.R., Moore, B.A. \& Bahrenburg, B. Oral delta-9tetrahydrocannabinol suppresses cannabis withdrawal symptoms. Drug and Alcohol Dependence 86, 22-29 (2007).

74. Rodriguez de Fonseca, F., Carrera, M.R., Navarro, M., Koob, G.F. \& Weiss, F. Activation of corticotropin-releasing factor in the limbic system during cannabinoid withdrawal. Science 276, 2050-4 (1997).

75. Caberlotto, L., Rimondini, R., Hansson, A., Eriksson, S. \& Heilig, M. Corticotropin-releasing hormone (CRH) mRNA expression in rat central amygdala in cannabinoid tolerance and withdrawal: evidence for an allostatic shift? Neuropsychopharmacology 29, 15-22 (2004).

References 74 and 75 were the first to demonstrate $\Delta^{9}$-THC withdrawal is associated with increased levels of the stress neuropeptide CRF in the amygdala, which probably contributes to negative affective states and diminished brain reward function; this withdrawal-related effect is also common to many other classes of abused drugs, including nicotine, alcohol and opiates.

76. Zorrilla, E.P., Logrip, M.L. \& Koob, G.F. Corticotropin releasing factor: a key role in the neurobiology of addiction. Front Neuroendocrinol 35, 234-44 (2014).

77. Vandrey, R., Budney, A., Hughes, J. \& Liguori, A. A within-subject comparison of withdrawal symptoms during abstinence from cannabis, tobacco, and both substances. Drug and alcohol dependence 92, 48-54 (2008).

78. Justinova, Z., Goldberg, S.R., Heishman, S.J. \& Tanda, G. Self-administration of cannabinoids by experimental animals and human marijuana smokers. Pharmacol Biochem Behav 81, 285-99 (2005).

This comprehensive review considers the reinforcing effects and abuse liability of $\Delta^{\mathbf{9}}$-THC and related cannabinoids as investigated in both human and animal studies.

79. Freeman, T.P. et al. Just say 'know': how do cannabinoid concentrations influence users' estimates of cannabis potency and the amount they roll in joints? Addiction 109, 1686-1694 (2014).

80. van der Pol, P. et al. Cross-sectional and prospective relation of cannabis potency, dosing and smoking behaviour with cannabis dependence: an ecological study. Addiction 109, 1101-9 (2014).

81. Freeman, T. \& Winstock, A. Examining the profile of high-potency cannabis and its association with severity of cannabis dependence. Psychological medicine 45, 3181-3189 (2015).

82. Morgan, C.J., Freeman, T.P., Schafer, G.L. \& Curran, H.V. Cannabidiol attenuates the appetitive effects of Delta 9-tetrahydrocannabinol in humans smoking their chosen cannabis.

Neuropsychopharmacology 35, 1879-85 (2010).

83. Field, M., Marhe, R. \& Franken, I.H. The clinical relevance of attentional bias in substance use disorders. CNS Spectr 19, 225-30 (2014).

84. Hindocha, C. et al. Acute effects of delta-9-tetrahydrocannabinol, cannabidiol and their combination on facial emotion recognition: A randomised, double-blind, placebo-controlled study in cannabis users. European Neuropsychopharmacology 25, 325-334 (2015).

85. Haney, M. et al. Oral Cannabidiol does not Alter the Subjective, Reinforcing or Cardiovascular Effects of Smoked Cannabis. Neuropsychopharmacology: official publication of the American College of Neuropsychopharmacology (2015).

86. Braida, D., Iosue, S., Pegorini, S. \& Sala, M. Delta9-tetrahydrocannabinol-induced conditioned place preference and intracerebroventricular self-administration in rats. Eur J Pharmacol 506, 63-9 (2004).

87. Zangen, A., Solinas, M., Ikemoto, S., Goldberg, S.R. \& Wise, R.A. Two brain sites for cannabinoid reward. J Neurosci 26, 4901-7 (2006). 
88. Gardner, E.L. et al. Facilitation of brain stimulation reward by delta 9-tetrahydrocannabinol. Psychopharmacology (Berl) 96, 142-4 (1988).

89. Vlachou, S., Nomikos, G.G. \& Panagis, G. CB1 cannabinoid receptor agonists increase intracranial self-stimulation thresholds in the rat. Psychopharmacology (Berl) 179, 498-508 (2005).

90. Vlachou, S., Nomikos, G.G., Stephens, D.N. \& Panagis, G. Lack of evidence for appetitive effects of Delta 9-tetrahydrocannabinol in the intracranial self-stimulation and conditioned place preference procedures in rodents. Behav Pharmacol 18, 311-9 (2007).

91. Sanudo-Pena, M.C. et al. Endogenous cannabinoids as an aversive or counter-rewarding system in the rat. Neurosci Lett 223, 125-8 (1997).

92. Cheer, J.F., Kendall, D.A. \& Marsden, C.A. Cannabinoid receptors and reward in the rat: a conditioned place preference study. Psychopharmacology (Berl) 151, 25-30 (2000).

93. Winstock, A.R. \& Barratt, M.J. Synthetic cannabis: a comparison of patterns of use and effect profile with natural cannabis in a large global sample. Drug and alcohol dependence 131, 106-111 (2013).

94. Justinova, Z., Tanda, G., Redhi, G.H. \& Goldberg, S.R. Self-administration of delta9tetrahydrocannabinol (THC) by drug naive squirrel monkeys. Psychopharmacology (Berl) 169, 135-40 (2003).

95. Fattore, L., Cossu, G., Martellotta, C.M. \& Fratta, W. Intravenous self-administration of the cannabinoid CB1 receptor agonist WIN 55,212-2 in rats. Psychopharmacology (Berl) 156, 410-6 (2001).

96. Lecca, D., Cacciapaglia, F., Valentini, V. \& Di Chiara, G. Monitoring extracellular dopamine in the rat nucleus accumbens shell and core during acquisition and maintenance of intravenous WIN 55,212-2 self-administration. Psychopharmacology (Berl) 188, 63-74 (2006).

97. Parsons, L.H. \& Hurd, Y.L. Endocannabinoid signaling in reward and addiction. Nature Reviews Neuroscience (in revision) (2015).

98. Serrano, A. \& Parsons, L.H. Endocannabinoid influence in drug reinforcement, dependence and addiction-related behaviors. Pharmacol Ther 132, 215-41 (2011).

99. Ceccarini, J. et al. [18F] MK-9470 PET measurement of cannabinoid CB1 receptor availability in chronic cannabis users. Addiction biology (2014).

100. Breivogel, C.S. et al. Chronic delta9-tetrahydrocannabinol treatment produces a time-dependent loss of cannabinoid receptors and cannabinoid receptor-activated $\mathrm{G}$ proteins in rat brain. J Neurochem $\mathbf{7 3}$, 2447-59 (1999).

101. Dudok, B. et al. Cell-specific STORM super-resolution imaging reveals nanoscale organization of cannabinoid signaling. Nature neuroscience 18, 75-86 (2015).

This study employed nanoscale imaging and electrophysiological techniques to demonstrate greater CB1R expression and influence on perisomatically projecting versus dendriticallyprojecting GABAergic interneurons in the mouse hippocampus, and that persistent deficits in hippocampal LTP following chronic $\Delta^{9}$-THC exposure result from near complete loss of CB1R at somatic synapses.

102. Sim-Selley, L.J. et al. Prolonged recovery rate of CB1 receptor adaptation after cessation of long-term cannabinoid administration. Molecular pharmacology 70, 986-996 (2006).

103. Hoffman, A.F., Oz, M., Caulder, T. \& Lupica, C.R. Functional tolerance and blockade of long-term depression at synapses in the nucleus accumbens after chronic cannabinoid exposure. J Neurosci 23 , 4815-20 (2003).

104. Mato, S. et al. A single in-vivo exposure to delta 9THC blocks endocannabinoid-mediated synaptic plasticity. Nat Neurosci 7, 585-6 (2004).

References 103 and 104 were among the first to demonstrate that $\Delta^{9}$-THC exposure disrupts synaptic plasticity of nucleus accumbens neurons in rodents.

105. Schlosburg, J.E. et al. Inhibitors of endocannabinoid-metabolizing enzymes reduce precipitated withdrawal responses in THC-dependent mice. AAPS J 11, 342-52 (2009).

106. Castelli, M.P. et al. Dysregulation of the endogenous cannabinoid system in adult rats prenatally treated with the cannabinoid agonist WIN 55,212-2. Eur J Pharmacol 573, 11-9 (2007).

107. Morgan, C.J. et al. Cerebrospinal fluid anandamide levels, cannabis use and psychotic-like symptoms. The British Journal of Psychiatry 202, 381-382 (2013).

108. Muhl, D. et al. Increased CB2 mRNA and anandamide in human blood after cessation of cannabis abuse. Naunyn Schmiedebergs Arch Pharmacol 387, 691-5 (2014).

109. Bossong, M.G. et al. Further human evidence for striatal dopamine release induced by administration of $\Delta$ 9-tetrahydrocannabinol (THC): selectivity to limbic striatum. Psychopharmacology, 1-7 (2015).

110. Nutt, D.J., Lingford-Hughes, A., Erritzoe, D. \& Stokes, P.A. The dopamine theory of addiction: 40 years of highs and lows. Nature Reviews Neuroscience 16 (2015). 
111. Cheer, J.F., Wassum, K.M., Heien, M.L., Phillips, P.E. \& Wightman, R.M. Cannabinoids enhance subsecond dopamine release in the nucleus accumbens of awake rats. J Neurosci 24, 4393-400 (2004).

112. Chen, J., Marmur, R., Pulles, A., Paredes, W. \& Gardner, E.L. Ventral tegmental microinjection of delta 9-tetrahydrocannabinol enhances ventral tegmental somatodendritic dopamine levels but not forebrain dopamine levels: evidence for local neural action by marijuana's psychoactive ingredient. Brain Res 621, 65-70 (1993).

113. Tanda, G., Pontieri, F.E. \& Di Chiara, G. Cannabinoid and heroin activation of mesolimbic dopamine transmission by a common mu1 opioid receptor mechanism. Science 276, 2048-50 (1997).

114. Diana, M., Melis, M., Muntoni, A.L. \& Gessa, G.L. Mesolimbic dopaminergic decline after cannabinoid withdrawal. Proc Natl Acad Sci U S A 95, 10269-73 (1998).

115. Tanda, G., Loddo, P. \& Di Chiara, G. Dependence of mesolimbic dopamine transmission on delta9tetrahydrocannabinol. Eur J Pharmacol 376, 23-6 (1999).

116. Bloomfield, M.A. et al. Dopaminergic function in cannabis users and its relationship to cannabisinduced psychotic symptoms. Biol Psychiatry 75, 470-8 (2014).

117. Ghazzaoui, R. \& Abi-Dargham, A. Imaging dopamine transmission parameters in cannabis dependence. Prog Neuropsychopharmacol Biol Psychiatry 52, 28-32 (2014).

118. Manzanares, J. et al. Chronic administration of cannabinoids regulates proenkephalin mRNA levels in selected regions of the rat brain. Brain Res Mol Brain Res 55, 126-32 (1998).

119. Valverde, O. et al. Delta9-tetrahydrocannabinol releases and facilitates the effects of endogenous enkephalins: reduction in morphine withdrawal syndrome without change in rewarding effect. Eur $J$ Neurosci 13, 1816-24 (2001).

120. Braida, D., Pozzi, M., Parolaro, D. \& Sala, M. Intracerebral self-administration of the cannabinoid receptor agonist CP 55,940 in the rat: interaction with the opioid system. Eur J Pharmacol 413, 227-34 (2001).

121. Justinova, Z., Tanda, G., Munzar, P. \& Goldberg, S.R. The opioid antagonist naltrexone reduces the reinforcing effects of Delta 9 tetrahydrocannabinol (THC) in squirrel monkeys. Psychopharmacology (Berl) 173, 186-94 (2004).

122. Haney, M. et al. Naltrexone Maintenance Decreases Cannabis Self-Administration and Subjective Effects in Daily Cannabis Smokers. Neuropsychopharmacology (2015).

123. Delfs, J.M., Zhu, Y., Druhan, J.P. \& Aston-Jones, G. Noradrenaline in the ventral forebrain is critical for opiate withdrawal-induced aversion. Nature 403, 430-4 (2000).

124. Moranta, D., Esteban, S. \& Garcia-Sevilla, J.A. Chronic treatment and withdrawal of the cannabinoid agonist WIN 55,212-2 modulate the sensitivity of presynaptic receptors involved in the regulation of monoamine syntheses in rat brain. Naunyn Schmiedebergs Arch Pharmacol 379, 61-72 (2009).

125. Page, M.E., Oropeza, V.C. \& Van Bockstaele, E.J. Local administration of a cannabinoid agonist alters norepinephrine efflux in the rat frontal cortex. Neurosci Lett 431, 1-5 (2008).

126. Jentsch, J.D., Andrusiak, E., Tran, A., Bowers, M.B., Jr. \& Roth, R.H. Delta 9-tetrahydrocannabinol increases prefrontal cortical catecholaminergic utilization and impairs spatial working memory in the rat: blockade of dopaminergic effects with HA966. Neuropsychopharmacology 16, 426-32 (1997).

127. Carvalho, A.F. \& Van Bockstaele, E.J. Cannabinoid modulation of noradrenergic circuits: implications for psychiatric disorders. Prog Neuropsychopharmacol Biol Psychiatry 38, 59-67 (2012).

128. Coffey, C., Carlin, J.B., Lynskey, M., Li, N. \& Patton, G.C. Adolescent precursors of cannabis dependence: findings from the Victorian Adolescent Health Cohort Study. Br J Psychiatry 182, 330-6 (2003).

129. Hines, L.A. et al. Onset of opportunity to use cannabis and progression from opportunity to dependence: Are influences consistent across transitions? Drug and Alcohol Dependence (2016).

130. Hindocha, C. et al. Associations between cigarette smoking and cannabis dependence: A longitudinal study of young cannabis users in the United Kingdom. Drug and alcohol dependence 148, 165-171 (2015).

131. Chen, C.-Y., O’Brien, M.S. \& Anthony, J.C. Who becomes cannabis dependent soon after onset of use? Epidemiological evidence from the United States: 2000-2001. Drug and alcohol dependence 79, 11-22 (2005).

132. Verweij, K.J. et al. Genetic and environmental influences on cannabis use initiation and problematic use: a meta-analysis of twin studies. Addiction 105, 417-30 (2010).

133. Agrawal, A. \& Lynskey, M.T. Candidate genes for cannabis use disorders: findings, challenges and directions. Addiction 104, 518-32 (2009).

134. Uhl, G.R. et al. "Higher order" addiction molecular genetics: convergent data from genome-wide association in humans and mice. Biochem Pharmacol 75, 98-111 (2008).

135. Chatters, R. et al. Psychological and psychosocial interventions for cannabis cessation in adults: A systematic review. Addiction Research \& Theory, 1-18 (2015). 
136. Marshall K, G.L., Ali R, Le Foll B. Pharmacotherapies for cannabis dependence. Cochrane Database of Systematic Reviews, CD008940 (2014).

137. Mason, B.J. et al. A proof-of-concept randomized controlled study of gabapentin: effects on cannabis use, withdrawal and executive function deficits in cannabis-dependent adults.

Neuropsychopharmacology 37, 1689-1698 (2012).

138. Gray, K.M. et al. A double-blind randomized controlled trial of N-acetylcysteine in cannabisdependent adolescents. American Journal of Psychiatry 169, 805-812 (2012).

139. Levin, F.R. et al. Dronabinol for the treatment of cannabis dependence: a randomized, double-blind, placebo-controlled trial. Drug and alcohol dependence 116, 142-150 (2011).

140. Allsop, D.J. et al. Nabiximols as an agonist replacement therapy during cannabis withdrawal: a randomized clinical trial. JAMA psychiatry 71, 281-291 (2014).

141. Lutz, B., Marsicano, G., Maldonado, R. \& Hillard, C.J. The endocannabinoid system in guarding against fear, anxiety and stress. Nature Reviews Neuroscience 16, 705-718 (2015).

142. Murillo-Rodriguez, E. et al. The emerging role of the endocannabinoid system in the sleep-wake cycle modulation. Cent Nerv Syst Agents Med Chem 11, 189-96 (2011).

143. Weinstein, A.M. \& Gorelick, D.A. Pharmacological treatment of cannabis dependence. Curr Pharm Des 17, 1351-8 (2011).

144. Clapper, J.R., Mangieri, R.A. \& Piomelli, D. The endocannabinoid system as a target for the treatment of cannabis dependence. Neuropharmacology 56 Suppl 1, 235-43 (2009).

145. van der Pol, P. et al. Mental health differences between frequent cannabis users with and without dependence and the general population. Addiction 108, 1459-1469 (2013).

146. Degenhardt, L., Hall, W. \& Lynskey, M. Testing hypotheses about the relationship between cannabis use and psychosis. Drug and alcohol dependence 71, 37-48 (2003).

147. Flórez-Salamanca, L. et al. Probability and predictors of cannabis use disorders relapse: results of the National Epidemiologic Survey on Alcohol and Related Conditions (NESARC). Drug and alcohol dependence 132, 127-133 (2013).

148. Christensen, R., Kristensen, P.K., Bartels, E.M., Bliddal, H. \& Astrup, A. Efficacy and safety of the weight-loss drug rimonabant: a meta-analysis of randomised trials. The Lancet 370, 1706-1713 (2007).

149. Nissen, S.E. et al. Effect of rimonabant on progression of atherosclerosis in patients with abdominal obesity and coronary artery disease: the STRADIVARIUS randomized controlled trial. Jama 299, 1547-1560 (2008).

150. Valverde, O. \& Torrens, M. CB1 receptor-deficient mice as a model for depression. Neuroscience 204, 193-206 (2012).

151. Zanelati, T.V., Biojone, C., Moreira, F.A., Guimaraes, F.S. \& Joca, S.R. Antidepressant-like effects of cannabidiol in mice: possible involvement of 5-HT1A receptors. Br J Pharmacol 159, 122-8 (2010).

152. Moreira, F.A. \& Wotjak, C.T. in Behavioral Neurobiology of Anxiety and Its Treatment 429-450 (Springer, 2010).

153. Sidhpura, N. \& Parsons, L.H. Endocannabinoid-mediated synaptic plasticity and addiction-related behavior. Neuropharmacology 61, 1070-1087 (2011).

154. Morgan, C. et al. Sub-chronic impact of cannabinoids in street cannabis on cognition, psychotic-like symptoms and psychological well-being. Psychological medicine 42, 391-400 (2012).

155. Buckner, J.D. \& Carroll, K.M. Effect of anxiety on treatment presentation and outcome: Results from the Marijuana Treatment Project. Psychiatry research 178, 493-500 (2010).

156. Van Dam, N.T., Bedi, G. \& Earleywine, M. Characteristics of clinically anxious versus non-anxious regular, heavy marijuana users. Addictive behaviors 37, 1217-1223 (2012).

157. Schafer, G. et al. Investigating the interaction between schizotypy, divergent thinking and cannabis use. Consciousness and cognition 21, 292-298 (2012).

158. Weiland, B.J. et al. Daily marijuana use is not associated with brain morphometric measures in adolescents or adults. The Journal of Neuroscience 35, 1505-1512 (2015).

159. Yücel, M. et al. The impact of cannabis use on cognitive functioning in patients with schizophrenia: a meta-analysis of existing findings and new data in a first-episode sample. Schizophrenia Bulletin 38, 316-330 (2012).

160. Hindocha, C., Freeman, T.P., Winstock, A.R. \& Lynskey, M.T. Vaping cannabis (marijuana) has the potential to reduce tobacco smoking in cannabis users. Addiction (2015).

161. Hasin, D.S. et al. Prevalence of Marijuana Use Disorders in the United States Between 2001-2002 and 2012-2013. JAMA psychiatry, 1-9 (2015).

162. Nutt, D.J., King, L.A. \& Nichols, D.E. Effects of Schedule I drug laws on neuroscience research and treatment innovation. Nature Reviews Neuroscience 14, 577-585 (2013).

163. Mokrysz, C. et al. Are IQ and educational outcomes in teenagers related to their cannabis use? A prospective cohort study. Journal of Psychopharmacology, doi: 10.1177/0269881115622241 (2016). 
164. Jackson, N.J. et al. Impact of adolescent marijuana use on intelligence: Results from two longitudinal twin studies. PNAS, doi: 10.1073/pnas.1516648113 (2016).

References 163 and 164 both examine associations between adolescent cannabis use and IQ by using data from large longitudinal cohorts in the United Kingdom and United States; both papers suggest there are non-causal explanations for the observed associations between adolescent cannabis use and lower IQ.

165. Temple, E.C., Brown, R.F. \& Hine, D.W. The 'grass ceiling': limitations in the literature hinder our understanding of cannabis use and its consequences. Addiction 106, 238-244 (2011).

An insightful review covering methodological inconsistencies in cananbis research, offering useful guidance for future research.

166. Fergusson, D.M., Horwood, L.J. \& Beautrais, A.L. Cannabis and educational achievement. Addiction 98, 1681-1692 (2003).

167. Silins, E. et al. Young adult sequelae of adolescent cannabis use: an integrative analysis. The Lancet Psychiatry 1, 286-293 (2014).

168. Lynskey, M.T. \& Hall, W. The effects of adolescent cannabis use on educational attainment: a review. Addiction 95, 1621-1630 (2000).

169. Townsend, L., Flisher, A.J. \& King, G. A systematic review of the relationship between high school dropout and substance use. Clinical Child and Family Psychology Review 10, 295-317 (2007).

170. Verweij, K.J., Huizink, A.C., Agrawal, A., Martin, N.G. \& Lynskey, M.T. Is the relationship between early-onset cannabis use and educational attainment causal or due to common liability? Drug and alcohol dependence 133, 580-586 (2013).

171. Bloomfield, M.A., Morgan, C.J., Kapur, S., Curran, H.V. \& Howes, O.D. The link between dopamine function and apathy in cannabis users: an [18F]-DOPA PET imaging study. Psychopharmacology 231, 2251-2259 (2014).

172. McCaffrey, D.F., Liccardo Pacula, R., Han, B. \& Ellickson, P. Marijuana use and high school dropout: the influence of unobservables. Health economics 19, 1281-1299 (2010).

173. Hooper, S.R., Woolley, D. \& De Bellis, M.D. Intellectual, neurocognitive, and academic achievement in abstinent adolescents with cannabis use disorder. Psychopharmacology 231, 1467-1477 (2014).

174. Stiby, A.I. et al. Adolescent Cannabis and Tobacco use and Educational Outcomes at Age 16: Birth Cohort Study. Addiction (2014).

175. Grant, J.D. et al. Associations of Alcohol, Nicotine, Cannabis, and Drug Use/Dependence with Educational Attainment: Evidence from Cotwin-Control Analyses. Alcoholism: Clinical and Experimental Research 36, 1412-1420 (2012).

176. Large, M., Sharma, S., Compton, M.T., Slade, T. \& Nielssen, O. Cannabis use and earlier onset of psychosis: a systematic meta-analysis. Arch Gen Psychiatry 68, 555-61 (2011).

177. Morgan, C.J.A., Freeman, T.P., Powell, J. \& Curran, H.V. AKT1 genotype moderates the acute psychotomimetic effects of naturalistically smoked cannabis in young cannabis smokers. Translational psychiatry 6, e738 (2016).

This is the largest study to date conducted on the acute response to cannabis and shows that psychotic-like symptoms in young healthy cannabis users are predicted by their $A K T 1$ genotype, replicating the same effect observed in schizophrenia (see Refs 178 and 179)

178. Di Forti, M. et al. Confirmation that the AKT1 (rs2494732) genotype influences the risk of psychosis in cannabis users. Biol Psychiatry 72, 811-6 (2012).

179. van Winkel, R., van Beveren, N.J., Simons, C., Genetic, R. \& Outcome of Psychosis, I. AKT1 moderation of cannabis-induced cognitive alterations in psychotic disorder. Neuropsychopharmacology 36, 2529-37 (2011).

180. Di Forti, M. et al. Proportion of patients in south London with first-episode psychosis attributable to use of high potency cannabis: a case-control study. The Lancet Psychiatry 2, 233-238 (2015).

The risk of individuals having a psychotic disorder was roughly three-fold higher in those saying they used skunk (which contains high concentrations of $\Delta^{9}$-THC and negligible CBD) and fivefold higher in those reporting daily skunk use, compared with non-users; hash (which has a lower $\Delta^{\mathbf{9}}$-THC content and more CBD) did not affect risk of a psychotic disorder.

181. Morgan, C.J. \& Curran, H.V. Effects of cannabidiol on schizophrenia-like symptoms in people who use cannabis. The British Journal of Psychiatry 192, 306-307 (2008).

The first study to show that psychosis-like symptoms are greater in those whose hair contains only $\Delta^{9}$-THC than in those whose hair contains both $\Delta^{9}$-THC and CBD or no cannabinoids suggesting a protective effect of CBD.

182. Leweke, F.M., Giuffrida, A., Wurster, U., Emrich, H.M. \& Piomelli, D. Elevated endogenous cannabinoids in schizophrenia. Neuroreport 10, 1665-9 (1999). 
183. Koethe, D. et al. Anandamide elevation in cerebrospinal fluid in initial prodromal states of psychosis. Br J Psychiatry 194, 371-2 (2009).

184. Di Marzo, V. Targeting the endocannabinoid system: to enhance or reduce? Nat Rev Drug Discov 7 , 438-55 (2008).

185. Volk, D.W. \& Lewis, D.A. The Role of Endocannabinoid Signaling in Cortical Inhibitory Neuron Dysfunction in Schizophrenia. Biological psychiatry (2015).

186. Association, A.P. Diagnostic and statistical manual of mental disorders, (DSM-5®) (American Psychiatric Pub, 2013).

187. Kandel, D.B. Stages and pathways of drug involvement: Examining the gateway hypothesis (Cambridge University Press, 2002).

188. Fergusson, D.M., Boden, J.M. \& Horwood, L.J. Cannabis use and other illicit drug use: testing the cannabis gateway hypothesis. Addiction 101, 556-569 (2006).

189. Lynskey, M.T. et al. Escalation of drug use in early-onset cannabis users vs co-twin controls. Jama 289, 427-433 (2003).

190. Cadoni, C., Simola, N., Espa, E., Fenu, S. \& Di Chiara, G. Strain dependence of adolescent Cannabis influence on heroin reward and mesolimbic dopamine transmission in adult Lewis and Fischer 344 rats. Addict Biol 20, 132-42 (2015).

191. Tomasiewicz, H.C. et al. Proenkephalin mediates the enduring effects of adolescent cannabis exposure associated with adult opiate vulnerability. Biol Psychiatry 72, 803-10 (2012).

192. Higuera-Matas, A. et al. Augmented acquisition of cocaine self-administration and altered brain glucose metabolism in adult female but not male rats exposed to a cannabinoid agonist during adolescence. Neuropsychopharmacology 33, 806-13 (2008).

193. Szutorisz, H. et al. Parental THC exposure leads to compulsive heroin-seeking and altered striatal synaptic plasticity in the subsequent generation. Neuropsychopharmacology 39, 1315-23 (2014).

194. MacCoun, R. \& Reuter, P. Evaluating alternative cannabis regimes. The British Journal of Psychiatry 178, 123-128 (2001).

195. Panlilio, L.V., Zanettini, C., Barnes, C., Solinas, M. \& Goldberg, S.R. Prior exposure to THC increases the addictive effects of nicotine in rats. Neuropsychopharmacology 38, 1198-1208 (2013).

196. Solinas, M., Panlilio, L. \& Goldberg, S. Exposure to delta-9-tetrahydrocannabinol (THC) increases subsequent heroin taking but not heroin's reinforcing efficacy: a self-administration study in rats. Neuropsychopharmacology: official publication of the American College of Neuropsychopharmacology 29, 1301-1311 (2004).

197. Panlilio, L.V., Solinas, M., Matthews, S.A. \& Goldberg, S.R. Previous exposure to THC alters the reinforcing efficacy and anxiety-related effects of cocaine in rats. Neuropsychopharmacology 32, 646657 (2007).

198. Patton, G.C., Coffey, C., Carlin, J.B., Sawyer, S.M. \& Lynskey, M. Reverse gateways? Frequent cannabis use as a predictor of tobacco initiation and nicotine dependence. Addiction 100, 1518-1525 (2005).

199. Carlezon, W.A., Jr. \& Thomas, M.J. Biological substrates of reward and aversion: a nucleus accumbens activity hypothesis. Neuropharmacology 56 Suppl 1, 122-32 (2009).

200. Glass, M., Dragunow, M. \& Faull, R.L. Cannabinoid receptors in the human brain: a detailed anatomical and quantitative autoradiographic study in the fetal, neonatal and adult human brain. Neuroscience 77, 299-318 (1997).

201. Wang, X., Dow-Edwards, D., Keller, E. \& Hurd, Y.L. Preferential limbic expression of the cannabinoid receptor mRNA in the human fetal brain. Neuroscience 118, 681-94 (2003).

202. Herkenham, M. et al. Characterization and localization of cannabinoid receptors in rat brain: a quantitative in vitro autoradiographic study. J Neurosci 11, 563-83 (1991).

203. Egertova, M. \& Elphick, M.R. Localisation of cannabinoid receptors in the rat brain using antibodies to the intracellular C-terminal tail of CB. J Comp Neurol 422, 159-71 (2000).

204. Tsou, K., Brown, S., Sanudo-Pena, M.C., Mackie, K. \& Walker, J.M. Immunohistochemical distribution of cannabinoid CB1 receptors in the rat central nervous system. Neuroscience 83, 393-411 (1998).

205. Hoffman, A.F. \& Lupica, C.R. Direct actions of cannabinoids on synaptic transmission in the nucleus accumbens: a comparison with opioids. J Neurophysiol 85, 72-83 (2001).

206. Pistis, M., Muntoni, A.L., Pillolla, G. \& Gessa, G.L. Cannabinoids inhibit excitatory inputs to neurons in the shell of the nucleus accumbens: an in vivo electrophysiological study. Eur J Neurosci 15, 1795802 (2002).

207. Tzavara, E.T., Wade, M. \& Nomikos, G.G. Biphasic effects of cannabinoids on acetylcholine release in the hippocampus: site and mechanism of action. J Neurosci 23, 9374-84 (2003). 
208. Pisanu, A., Acquas, E., Fenu, S. \& Di Chiara, G. Modulation of Delta(9)-THC-induced increase of cortical and hippocampal acetylcholine release by micro opioid and $\mathrm{D}^{1}$ dopamine receptors.

Neuropharmacology 50, 661-70 (2006).

209. Pistis, M. et al. Delta(9)-tetrahydrocannabinol decreases extracellular GABA and increases extracellular glutamate and dopamine levels in the rat prefrontal cortex: an in vivo microdialysis study. Brain Res 948, 155-8 (2002).

210. Higuera-Matas, A. et al. Periadolescent exposure to cannabinoids alters the striatal and hippocampal dopaminergic system in the adult rat brain. Eur Neuropsychopharmacol 20, 895-906 (2010).

211. Rubino, T. et al. Chronic delta 9-tetrahydrocannabinol during adolescence provokes sex-dependent changes in the emotional profile in adult rats: behavioral and biochemical correlates. Neuropsychopharmacology 33, 2760-71 (2008).

212. Ellgren, M., Spano, S.M. \& Hurd, Y.L. Adolescent cannabis exposure alters opiate intake and opioid limbic neuronal populations in adult rats. Neuropsychopharmacology 32, 607-15 (2007).

213. Morel, L.J., Giros, B. \& Dauge, V. Adolescent exposure to chronic delta-9-tetrahydrocannabinol blocks opiate dependence in maternally deprived rats. Neuropsychopharmacology 34, 2469-76 (2009).

\section{Box 1. Does cannabis affect IQ or educational attainment?}

Case-control and prospective cohort studies have found associations between cannabis use and both lower IQ and lower educational attainment. But do these associations reflect any causal relationships?

\section{Does cannabis affect IQ?}

To date, there have been three large prospective cohort studies that have assessed the relationship between cannabis use and IQ.

In a New Zealand birth cohort study of 1,037 38-year-olds born in 1972 or 1973, persistent cannabis dependence was associated with a decline of up to 6 IQ points from that measured at age $7-13$ years $^{54}$. The decline was particularly evident for those who developed cannabis dependence in adolescence, and remained apparent even for those who, at age 38 years, used cannabis less than once a week.

By contrast, a UK birth cohort study of 2,235 15-16-year-old adolescents born in 1991 or 1992 found that cumulative cannabis use was not associated with a lower IQ compared with nonusing controls, once IQ measured pre-teen and various potential confounders (in particular, the adolescents' use of cigarettes and alcohol) were accounted for ${ }^{163}$. Cannabis use was relatively low in this study, with only 72 adolescents reporting more than 50 lifetime cannabis exposures.

A US prospective cohort study of 3,066 17-20-year-olds found no difference in IQ change from that measured at age 9-12 years between monozygotic and dizygotic twins discordant for 
cannabis use ${ }^{164}$. However, there were only 47 discordant twin pairs in which the cannabis-using twin had used cannabis frequently (more than 30 cumulative uses, and/or daily use), limiting the strength of any conclusions from this study.

The UK and US studies therefore both suggest that genetic or environmental factors drive the observed associations between lower IQ and cannabis use, although both cohorts included younger participants with fewer cannabis exposures than did the New Zealand study.

To date, all studies have relied on retrospective self-report of cannabis use, have ignored possible residual effects of the drug on IQ test performance and have not addressed the potency or variety of cannabis used (see main article). Addressing these issues with confirmation of exposure to $\Delta^{9}$-THC and other cannabinoids (such as CBD) using hair samples from participants may lead to more reliable and consistent findings ${ }^{165}$.

\section{Does cannabis use affect educational attainment?}

Several case-control and longitudinal studies have provided fairly consistent evidence of associations between adolescent cannabis use and both early school leaving and poorer educational performance ${ }^{166-169}$. But the mechanisms producing these relationships remain hotly debated ${ }^{170}$.

Causal explanations have posited that heavy cannabis use results in cognitive and/or motivational deficits, which in turn result in poorer educational attainment. Although there are many anecdotes about an 'amotivational syndrome' resulting from heavy cannabis use, a recent positron emission tomography study demonstrated that cannabis users had reductions in striatal dopamine synthesis that correlated with a measure of amotivation ${ }^{171}$.

Alternatively, reverse causality has been also suggested; that is, perhaps poorer educational attainment leads to cannabis use ${ }^{166,168}$. However, the one study that addressed this hypothesis showed that the association between early school leaving and later cannabis use could be accounted for by cannabis use before leaving school early ${ }^{166}$.

The other alternative is that educational attainment and cannabis use may not be causally related but instead share common risk factors ${ }^{168,170,172}$. Reported associations between cannabis use and lower educational attainment have typically been robust to adjustment for some potential confounders such as early-life factors, baseline school performance or cognitive ability, social disadvantage and parental educational achievement) ${ }^{167,169}$. However, the 
potential role of teenage behaviours that typically occur alongside cannabis use — including use of other substances and other 'risky' behaviours such as truancy - remain relatively unexplored $^{163,170 .}$

Recent analyses showed that adjusting for teenage use of other substances attenuated the association between cannabis use and school attainment ${ }^{163,173,174}$. As such, the existence of unmeasured confounds is often posited to account for the negative associations with cannabis $163,168,170$. Indeed, this idea is strongly supported by recent genetic studies that found no difference in early school leaving ${ }^{170}$ or years of education ${ }^{175}$ between both monozygotic and dizygotic twin pairs who were discordant for cannabis use ${ }^{170,175}$.

\section{Box 2 | Cannabis and psychosis: cause, consequence or correlation?}

Nearly 2,000 studies have been published on this topic since 1962, and the pro-psychotic effects of cannabis have dominated media reporting about this drug. But how clear is the link? A number of longitudinal, population-based studies show an earlier first episode ${ }^{176}$ and a roughly two-fold increase in risk of psychosis with regular cannabis use ${ }^{62}$. Yet the vast majority of people who use cannabis do not develop psychotic disorders such as schizophrenia, and many people diagnosed with such disorders have never used cannabis.

More agreement is found in evidence that heavy cannabis use may mean that young people who are vulnerable to psychosis develop the disorder when they may not have otherwise done so. Converging data suggest that this may have a genetic basis, with certain polymorphisms of the gene encoding AKT1 potentially conferring risk of psychosis following smoking cannabis acutely $^{177}$ and chronically ${ }^{178,179}$.

The type of cannabis used has recently been found to impact on risk of psychosis: self-reported hash use, even daily, is not associated with increased risk of psychosis, whereas self-reported daily use of skunk (which contains high levels of $\Delta^{9}$-THC and negligible amounts of CBD) is associated with a five-fold greater chance of having schizophrenia ${ }^{180}$. Several studies using objective biological markers of use have shown that CBD reduces the psychosis-like effects of $\mathrm{THC}^{154,181}$

How cannabis interacts with the brain to increase psychosis risk is unclear. One confusing finding is that of reduced dopamine release in cannabis users ${ }^{116}$, which seems inconsistent with 
the higher levels of dopamine release observed in people with psychosis. Disruptions in the brain's endocannabinoid system, on the other hand, have been found in psychosis and may provide clues as to the pro-psychotic impact of cannabis. Higher levels of anandamide (AEA) in the cerebrospinal fluid have been associated with lower psychotic symptoms in individuals diagnosed with schizophrenia ${ }^{182}$, in individuals classified as having prodromal schizophrenia ${ }^{183}$ who do not smoke cannabis and in cannabis users without a diagnosis of schizophrenia ${ }^{107}$. AEA is known to have a neuromodulatory role in the brain; thus, during prodromal or first-episode psychosis, AEA may be increased to attempt to control dysregulated brain dopamine ${ }^{184}$.

In addition, in vivo positron emission tomography imaging and postmortem receptor autoradiography studies have consistently reported higher levels of ligand binding to cannabinoid 1 receptors in several cortical regions of people with schizophrenia ${ }^{185}$. Whether these alterations are part of the disease pathology or a compensatory response remains unclear, but they do suggest a molecular basis for a heightened sensitivity to cannabis of individuals with schizophrenia, and perhaps of those at risk.

\section{Box 3: 'Cannabis use disorder'}

Cannabis related problems vary on a continuum and it is important to define these not only for clinical diagnosis and treatment but also for research. The Diagnostic Statistical Manual Edition, fifth edition (DSM-5) ${ }^{186}$ has combined the definitions for 'cannabis abuse' and 'cannabis dependence' to provide criteria for the diagnosis of 'cannabis use disorder' (CUD). DSM-5 states that CUD is: "A problematic pattern of cannabis use leading to clinically significant impairment or distress, as manifested by at least 2 of the [symptoms from the three lists below], occurring within a 12-month period ${ }^{186}$ ". Mild CUD is associated with having 2 or 3 of these symptoms, moderate CUD, with 4 or 5 symptoms, and severe CUD, with 6 or more symptoms.

One advantage of combining abuse and dependence criteria in CUD is to provide a clearer continuum between mild and severe, because previously all cases of dependence also met criteria for abuse. Another advantage is that dependence is often a normal bodily response to a substance (for example, a prescribed pain killer) which should not to be confused with addiction (insomuch as stopping use of that pain killer does not necessarily lead to drugseeking). 
The following symptoms were included from $D S M-I V$ criteria for cannabis dependence:

- Cannabis is often taken in larger amounts or over a longer period than was intended

- There is a persistent desire or unsuccessful efforts to cut down or control cannabis use

- A great deal of time is spent in activities necessary to obtain cannabis, use cannabis, or recover from its effects

- Important social, occupational, or recreational activities are given up or reduced because of cannabis use

- Cannabis use is continued despite knowledge of having a persistent or recurrent physical or psychological problem that is likely to have been caused or exacerbated by cannabis

- Tolerance, as defined by either [1] a need for markedly increased cannabis to achieve intoxication or desired effect or [2] a markedly diminished effect with continued use of the same amount of the substance

- Withdrawal, as manifested by either [1] the characteristic withdrawal syndrome for cannabis or [2] cannabis is taken to relieve or avoid withdrawal symptoms

The following symptoms were included from DSM-IV criteria for cannabis abuse:

- Recurrent cannabis use resulting in a failure to fulfil major role obligations at work, school, or home

- Continued cannabis use despite having persistent or recurrent social or interpersonal problems caused or exacerbated by the effects of cannabis

- Recurrent cannabis use in situations in which it is physically hazardous

This item was added as a new symptom to DSM-5:

- Craving, or a strong desire or urge to use cannabis

One DSM-IV criterion - concerning persistent legal problems related to cannabis use - was dropped and not included in DSM-5 because it largely reflected sociocultural factors to do with policing and law enforcement rather than cannabis use factors. 


\section{Box 4: The gateway theory}

Although cannabis is traditionally considered as a 'soft' drug, it is widely believed to act as a 'gateway' to harder drugs such as cocaine or heroin (with harm defined in terms of detrimental effects of using that substance on the individual and on society). According to this theory, there is a sequential progression from one drug to the next (for example, cannabis leading to cocaine, and then heroin) ${ }^{187}$. This theory rests on evidence that use of one drug increases the likelihood of using the next drug in the 'sequence', which may be interpreted as causal if all other confounds are accounted for ${ }^{187}$.

Evidence does support sequence and association between cannabis and other illicit drugs, and these effects increase with frequency of cannabis use and adolescent onset ${ }^{188}$. Additionally, a twin study ${ }^{189}$ suggested that these effects cannot be attributed to shared genetic or environmental factors alone. Studies in rats demonstrate that adolescent $\Delta^{9}$-THC exposure results in increased opiate consumption and facilitated learning of cocaine self-administration in adulthood ${ }^{190-192}$, and that the influence of adolescent $\Delta^{9}$-THC exposure on opiate reward may be transgenerational ${ }^{193}$.

Establishing causality remains challenging, however, and putative mechanisms remain speculative. For example, one possibility is that the wide availability of cannabis means people are more likely to use it first and this increases the chances that other illicit drugs are used because of contact with other drug users and with people selling illicit drugs ${ }^{189}$. This formed part of The Netherlands' rationale for the regulated sale of cannabis; incidentally, the rates of cocaine use among people who have used cannabis are lower there $(22 \%)$ than in the United States $(33 \%)^{194}$.

Biological explanations for the gateway hypothesis are supported by evidence that exposure to THC enhances the reinforcing effects of other drugs. Intriguingly, however, prior $\Delta^{9}$-THC ingestion increases rats' self-administration of nicotine and the reinforcing effects of nicotine ${ }^{195}$, but this does not occur with prior cocaine or heroin exposure ${ }^{196,197}$. Indeed, one of the most potentially harmful and underappreciated effects of cannabis is the 'reverse gateway': by smoking tobacco together with cannabis in 'joints', individuals may progress to nicotine addiction $^{160,198}$. 
Figure 1 | Cannabinoid 1 receptor distribution within reward-, habit- and cognitionrelated circuits. A simplified conceptualization of the major circuits implicated in reward (namely, the ventral tegmental area (VTA), nucleus accumbens ${ }^{211}$ and ventral pallidum $(\mathrm{VP}))^{65,66,199}$, stimulus-response habit formation (the dorsolateral striatum (dlStr) and globus pallidus (GP) $)^{67}$ and cognition (the prefrontal cortex (PFC), hippocampus (HIPP), and amygdalar regions). Among these regions, CB1Rs are expressed with the following order of density ${ }^{200-204}$ : HIPP $\approx$ basolateral amygdala $\left.(\mathrm{BLA}) \approx \mathrm{PFC}>\mathrm{VP} \approx \mathrm{GP}\right) \approx \mathrm{DSTr}>\mathrm{NAc}>\mathrm{VTA}$ $\approx$ bed nucleus of the stria terminalis $(\mathrm{BNST})>$ central nucleus of the amygdala $(\mathrm{CeA})$. Within the amygdala, CB1R expression is highest in the lateral and basolateral nuclei, with substantially lower expression in the central nucleus ${ }^{203}$. In the dorsal striatum there is a comparable medial-lateral gradient of CB1R expression with greater levels of expression evident in lateral aspects, and comparatively lesser CB1R expression is observed in the NAc ${ }^{204}$. The dense CB1R expression in HIPP, PFC and amygdalar regions underlies the effects of cannabis and CB1R agonists on cognitive and memory function, whereas the CB1R presence within the mesocorticolimbic regions (VTA, NAc, PFC) contribute to the rewarding effects produced by cannabinoids.

Figure 2 | Effects of acute or chronic exposure to cannabis on reward- and cognitionrelated circuits. Graphical summary of alterations in neurobiological function resulting from chronic cannabinoid agonist exposure based on studies in rodents, non-human primates and humans. These collected observations should be viewed as a model of chronic cannabis exposure, rather than an explicit representation of cannabis-induced alterations in human brain function. a Acute cannabis or moderate-dose CB1R agonist exposure induces neurochemical events in the mesolimbic system similar to those produced by other drugs of abuse, including increased dopamine (DA) release and an attenuation of evoked GABA and glutamate (Glu) release in the nucleus accumbens 109,111-113, 205, 206. The induced increase in opioid peptide release in the NAc ${ }^{118,119}$ probably also contributes to the acute rewarding effects of $\Delta^{9}$-THC. Disruptions in cognitive function (including attention and memory impairments) probably result from: decreased acetylcholine (ACh) release in hippocampus (HIPP) and prefrontal cortex $(\mathrm{PFC})^{207,208}$; reduced GABA release and increased Glu release in the $\mathrm{PFC}^{209}$; and increased noradrenaline (NA) release in HIPP and frontal cortical areas ${ }^{124-126}$. b | Chronic $\Delta^{9}$ THC or CB1R agonist exposure results in decreased CB1R expression and function in many brain regions. Positron emission tomography imaging of daily cannabis users has revealed considerable decreases in CB1R levels, particularly in cortical regions ${ }^{30,31}$, that correlate with 
years of cannabis use and withdrawal, and rapidly normalise during abstinence ${ }^{30,31}$. Rodent studies demonstrate regional differences in the effects of chronic $\Delta^{9}$-THC on CB1R expression and function, with rapid and profound decreases evident in the hippocampus and layer VI of frontal cortex, smaller but still statistically significant decreases in striatal and amygdalar regions, and nonsignificant disruptions in regions such as the globus pallidus (GP) and hypothalamic nuclei ${ }^{100,101}$. The pronounced deficits in cortical and hippocampal CB1R function are consistent with memory and cognitive impairments associated with chronic cannabis use in humans. In this regard, chronic adolescent $\Delta^{9}$-THC exposure results in persistent disruptions of the hippocampal and cortical signalling that is critical for proper memory and cognitive functions. Chronic $\Delta^{9}$-THC exposure also disrupts reward-related signalling mechanisms in the mesolimbic system by reducing DA cell density in the ventral tegmental area $(\mathrm{VTA})^{210}$ and by decreasing VTA DA cell firing and DA release in the NAc during both spontaneous and CB1R antagonist-precipitated withdrawal ${ }^{114,115}$. Consistent with these observations in animal models, reduced striatal DA synthesis has been observed in human cannabis users, and this effect appears to be driven by individuals meeting cannabis use disorder criteria ${ }^{116}$. Together with increased stress-related signalling, such as dynorphin release in the NAc $\mathrm{N}^{211}$ and corticotropin releasing factor release in the amygdala ${ }^{74,75}$, these deficits in mesostriatal DA function may contribute to negative affective states associated with THC abstinence. Adolescent $\Delta^{9}$-THC exposure also results in persistent increases in opioid peptide gene expression and influences the mesolimbic system of rats ${ }^{191,212,213}$, possibly contributing to an increase in the rewarding effects of opiates and increase opiate by these animals in adulthood $^{190-192,212}$.

\section{GLOSSARY}

\section{Cannabis abuse}

Cannabis use that is problematic for various aspects of an individual's life, (for example, causing occupational, educational, or social problems), or that is carried out in dangerous contexts.

\section{Cannabis dependence}

A group of severe consequences of repeated cannabis use, including tolerance to effects, withdrawal symptoms upon cessation, dysregulation of use, increased involvement with cannabis at the expense of other activities, and continued use despite the problems it causes.

\section{Conditioned place-preference (CPP) procedure}

Pavlovian conditioning procedure used to index the motivational properties of drug experience. Typically, the different in time spent in an environment associated with drug intoxication is compared with that spent in a neutral context. 


\section{Episodic memory}

Personal, contextualised autobiographical memory of past experiences.

\section{Intracranial self-stimulation (ICSS)}

An operant paradigm in which animals perform a behavioural response to receive brief electrical pulses into specific regions in the brain reward pathways.

\section{Working memory}

The capacity to hold information 'online' (maintenance) and manipulate it.

\section{Long-term depression}

(LTD). An enduring decrease in the strength of neurotransmission at a synapse, implicated learning and memory.

\section{Long-term potentiation}

(LTP). An lasting increase in the strength of neurotransmission at a synapse, implicated in learning and memory.

\section{Psychosis}

A mental disturbance characterized by aberrant perceptions (hallucinations) and thoughts (delusions) that causes an individual to lose touch with external reality.

\section{Reinforcement}

A learning process through which particular stimuli or events (such as familiar drug-taking environments, or pleasant drug effects) influence the likelihood or strength of behaviour, such as drug seeking.

\section{Biographies}

H. Valerie Curran is currently Professor of Psychopharmacology at University College London, Director of the UCL Clinical Psychopharmacology Unit (CPU) and Clinical Research Lead at local London Drug Services. Her research, mainly funded by the UK Medical Research Council, focuses on: neurobiological, neurocognitive and emotional factors in substance use disorders; the development of novel treatments for addictions; and individual resilience and vulnerability factors in substance use disorders. The CPU website is: http://www.ucl.ac.uk/cpu

Tom P. Freeman is a postdoctoral research associate at University College London where he is coordinating a clinical trial for the treatment of cannabis addiction, funded by the UK Medical Research Council. His research interests include: contrasting the harmful and 
beneficial effects of different cannabinoids; the relationship between addiction and psychosis; and using experimental medicine to inform novel treatments for mental health problems.

Claire Mokrysz is currently a UK Medical Research Council Ph.D. student in psychopharmacology at University College London. Her interests include the neurocognitive effects of recreational drug use and the identification of vulnerability factors associated with an increased risk of drug-related harm. Her research currently focuses on whether adolescence represents a period of heightened vulnerability to the potential harms of cannabis and alcohol use.

David A. Lewis is the Thomas Detre Professor and Chair of the Department of Psychiatry at the University of Pittsburgh. His research interests focus on the functional architecture and development of the primate prefrontal cortex, and the alterations in this circuitry associated with schizophrenia and adolescent cannabis exposure.

Celia J. A. Morgan is Professor of Psychopharmacology at the University of Exeter. Her work focuses on translational approaches to the discovery of new pharmacological and psychological treatments for addiction and other psychiatric disorders. The UK Medical Research Council funds her main research.

Loren H. Parsons received his Ph.D. in chemistry from Emory University in Atlanta, Georgia, USA followed by postdoctoral training in behavioural pharmacology and neurochemistry at The Scripps Research Institute (TSRI) in La Jolla, California, USA. He is currently Professor in the Committee on the Neurobiology of Addictive Disorders at TSRI, Director of the TSRI Alcohol Research Center and Director of the Ruth L. Kirchstein Training Program at TSRI. His research looks into the neurobiological bases of addiction, with particular focus on the role of dysregulated endocannabinoid signaling in drug dependence and protracted withdrawal, and the cortical mechanisms underlying dependence-related cognitive impairment. The Parsons lab website is: http://www.scripps.edu/parsons/Research.html.

\section{Key Points:}

- Cannabis contain over 100 unique ingredients we call 'cannabinoids', and the proportions of these vary widely across different strains of the plant. High-delta-9tetrahydrocannabinol ( $\triangle 9$-THC) varieties with negligible levels of cannabidiol (CBD) now dominate many Western markets and are more harmful than lower- $\Delta 9-\mathrm{THC}$, higher-CBD varieties. 
- Like other recreational drugs, $\triangle 9$-THC increases release of dopamine and opioid peptides (in preclinical studies) and alters endocannabinoid processing in the mesocorticolimbic reward system. Long-term $\Delta 9$-THC exposure leads to a downregulation of brain cannabinoid receptor function that reverses following abstinence.

- People who try cannabis are nine-fold more likely to become addicted to it than to develop psychosis. Cannabis addiction is an increasing problem globally and no effective pharmacological treatments currently exist - this remains a major unmet clinical need.

- The association between cannabis use and psychosis can be influenced by a number of vulnerability factors, including genetics, environmental factors and the frequency and type of cannabis used. Evidence linking cannabis use with the development of depression and anxiety is less consistent, although these disorders are often comorbid with cannabis addiction.

- The acute effects of cannabis upon cognitive function are well documented and the most robust, dose-related decrements are to working and episodic memory. Its longterm cognitive effects remain controversial, are influenced by many confounds and appear to subside a month after stopping use of the drug.

- We should ensure that global legislative changes are informed by neuroscience and public health. They should mitigate against adolescent uptake and the availability of highly potent products, including synthetic agents such as 'spice', that act as full cannabinoid receptor agonists.

The authors declare competing interests: see Web version for details. 
identification and validation and new compound development for Autifony, Bristol-Myers Squibb, Concert Pharmaceuticals and Sunovion. There are no other conflicts of interest to declare. [Au: Edits OK?]

Figure 1

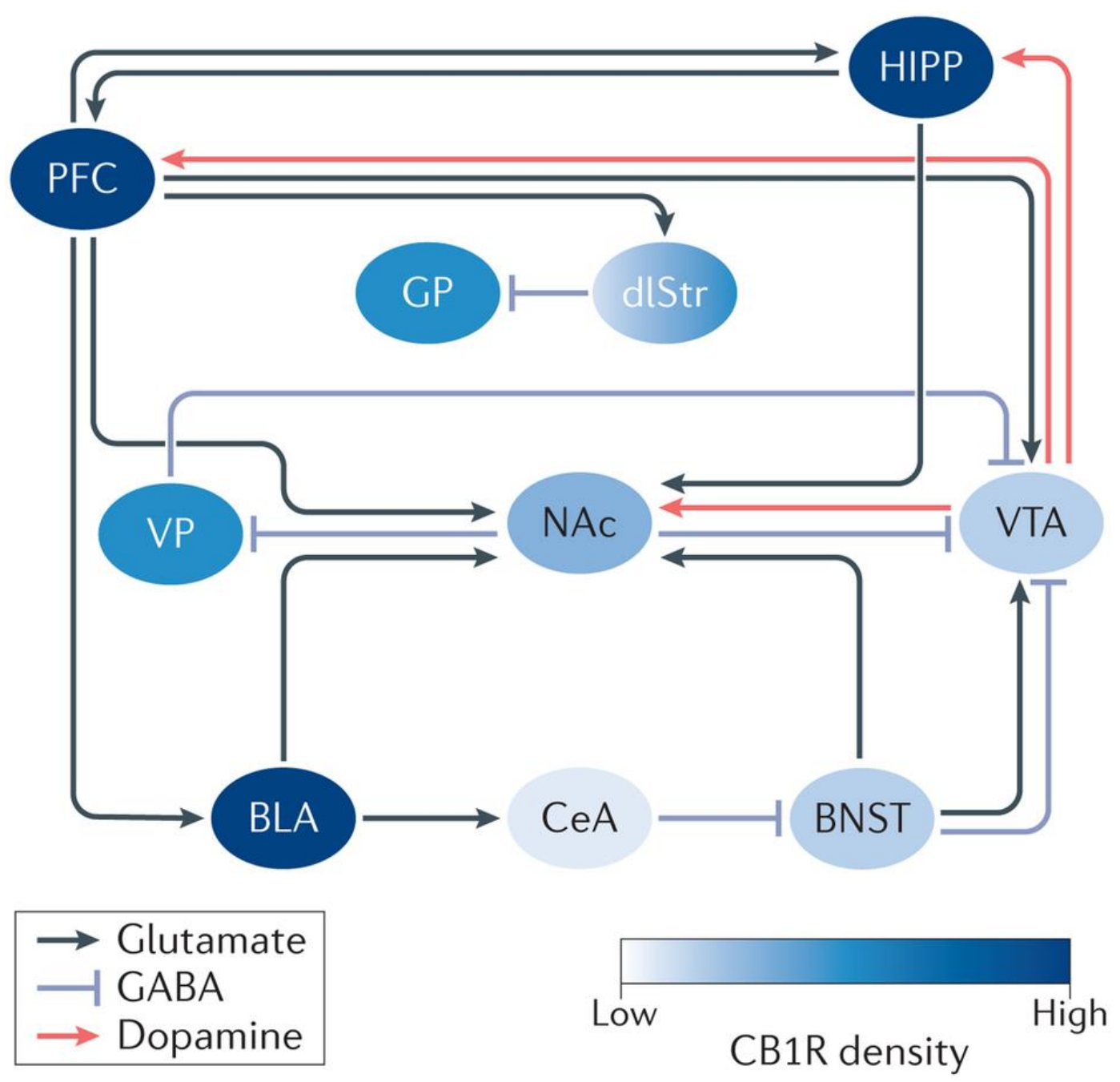

Nature Reviews | Neuroscience 
Figure 2
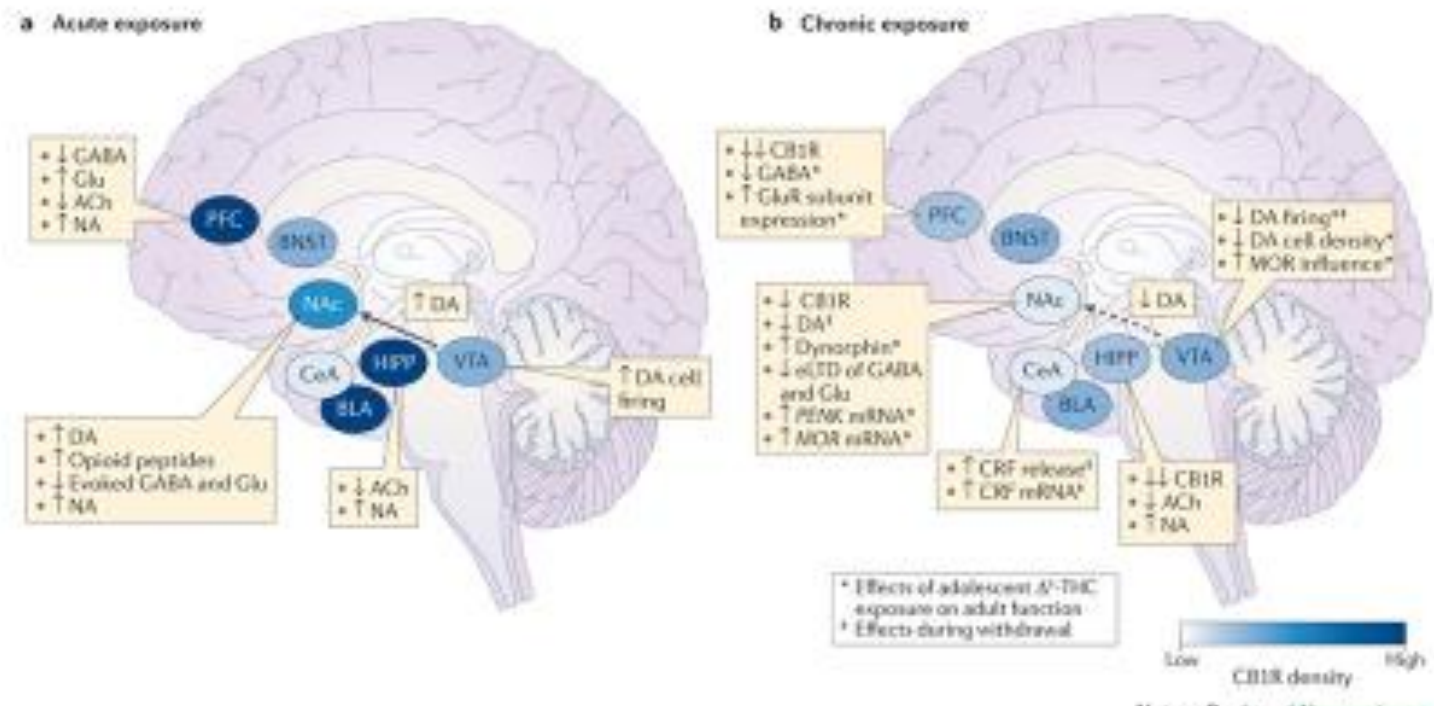

Nature Rerieme i Neuroncience 